\title{
Molecular-statistical theory of elasticity in nematic liquid crystals composed of polar and nonpolar molecules
}

\author{
M. A. Osipov, ${ }^{1,2}$ A. A. Antonov $\odot,{ }^{3}$ and M. V. Gorkunov $\odot^{3}$ \\ ${ }^{1}$ Department of Mathematics and Statistics, University of Strathclyde, Glasgow G1 1XH, Scotland, United Kingdom \\ ${ }^{2}$ Topchiev Institute of Petrochemical Synthesis, Russian Academy of Sciences, 119991 Moscow, Russia \\ ${ }^{3}$ Shubnikov Institute of Crystallography, Federal Scientific Research Centre "Crystallography and Photonics," \\ Russian Academy of Sciences, 119333 Moscow, Russia
}

(Received 7 August 2020; revised 6 February 2021; accepted 7 April 2021; published 3 May 2021)

\begin{abstract}
A molecular-statistical theory of the orientational elasticity of nematic liquid crystals has been developed employing the orientational deformation tensor which describes the rotation of the director. An explicit expression for the general elasticity tensor of the nematic phase has been obtained and the Frank elastic constants are expressed in terms of the three independent parameters of this tensor. Explicit expressions for the Frank elastic constants have been derived in the molecular field approximation in terms of the orientational order parameters and the corresponding coefficients of expansion of the intermolecular potential in spherical invariants. Frank elastic constants have been calculated numerically for nematic liquid crystals composed of both polar and nonpolar molecules together with the orientational order parameters using the classical Gay-Berne model interaction potential and the two of its popular modifications. The polarity of the uniaxial molecular shape has been directly introduced into the model potential by modifying the distance of closest approach. The elastic constants are presented as functions of temperature for different values of the molecular elongation, the anisotropy of the potential well and the molecular shape polarity. It has been shown that the elastic constants are much more sensitive to the details of the intermolecular interaction potential in comparison with the orientational order parameters. In particular, a relatively weak polarity of the molecular shape may result in an unusual decrease of the splay constant $K_{11}$ which may vanish at some temperature leading to the instability of the homogeneous nematic phase. This may represent a mechanism of the formation of the splay-bend phase.
\end{abstract}

DOI: 10.1103/PhysRevE.103.052701

\section{INTRODUCTION}

In nematic liquid crystals (LCs) primary axes of anisotropic molecules are partially ordered along the local symmetry axis specified by the director (a unit vector) $\mathbf{n}$ or, more precisely, along the principal axis of the uniaxial tensor $n_{\alpha} n_{\beta}-\delta_{\alpha \beta} / 3$. The equilibrium distribution of the director may be inhomogeneous under the action of external fields or boundary condition and in such cases the inhomogeneous nematic LC possesses orientational deformation energy specified by three elastic constants $K_{11}, K_{22}$, and $K_{33}$ which correspond to the splay, twist, and bend deformations, respectively $[1,2]$. The elastic constants of nematic LCs are very important material parameters as they determine the response of the LC to external fields which is crucial for LC display devices and numerous other LC applications. On the other hand, the elastic constants are very sensitive to the microscopic state of a nematic LC and to the intermolecular interaction potential which is determined by the structure of mesogenic molecules.

Since the later 2000s, the orientational elasticity of nematic LCs has attracted a renewed attention mainly due to the discovery of the anomalous behavior of Frank elastic constants in bent-core LCs. A dramatic reduction of the bend elastic constant has been observed in many bent-core nematics and in particular close to the transition into the twist-bend nematic phase [3-5]. The twist elastic constant is also significantly reduced in some flexible bent-core materials [6]. A number of attempts have been made to explain such an anomalous behavior by using a continuum theory [7] and a molecular theory of nematic elasticity generalized to the case of biaxial and polar molecules [8,9], although the existing theory is far from being complete.

One notes that the continuum theory of nematic elasticity is very well established and the corresponding molecular theory for LCs composed of uniaxial molecules has been developed in the past by many authors (see Refs. [10-20] and a review [21]). In a number of theories, only steric repulsion between hard spherocylinders or hard ellipsoids of revolution has been taken into account using the Onsager theory $[11,12,19]$, density-functional approach $[17,22]$ or the reference system of parallel hard ellipsoids $[13,15]$. The elastic constants have been expressed in terms of the geometrical parameters of the model molecular shape. Priest [11] has obtained rather general expressions for the elastic constants in terms of the secondand fourth-order orientational order parameters employing the spherical harmonics expansion of the general pair interaction potential and the pair correlation function. In other molecular theories, both intermolecular repulsion and attraction have been taken into consideration including the dispersion interaction between molecules with anisotropic polarizability 
$[10,16,17,23]$ and electrostatic interactions between permanent molecular dipoles and quadrupoles [20].

In this paper, we propose a general approach to deriving the free energy density of distorted nematic LCs based on the so-called orientational deformation tensor $\gamma_{i j}$ which explicitly describes the director rotation. In this approach, the elasticity tensor is explicitly expressed as a finite sum of simple terms, and all elastic constants are presented in terms of the three relevant coefficients of such a representation. Employing the molecular field approximation together with the expansion of pair interaction potential in terms of the so-called spherical invariants [24,25], we derive general expressions for the elastic constants. In contrast to the general expressions for the elastic constants derived by Poniewierski and Stecki [22], the expressions presented in this paper are independent of the derivatives of the single-molecule distribution function and are presented as the ensemble averages of the corresponding quantities. Thus the theory establishes a direct relationship between the intermolecular potential and Frank elastic constants. The corresponding general theory of elasticity in nematic LCs is presented in Secs. II and III. In Sec. IV, for illustration, we expand both the classical Gay-Berne (G-B) model intermolecular interaction potential and the two popular modifications in spherical invariants and employ the expansion coefficients to numerically evaluate the orientational order parameters and Frank elastic constants for nonpolar molecules. The calculated elastic constants are presented as functions of the temperature for different values of molecular anisometry and anisotropy of the potential well parametrizing the G-B potentials. In Sec. VI we generalize the theory of nematic elasticity to the case of polar molecules and numerically calculate the corrections to the splay and bend elastic constants which are determined by polar molecular shape. Finally in Sec. VII we discuss the results of this study.

\section{FREE ENERGY OF THE DISTORTED NEMATIC PHASE}

The distortion free energy is traditionally derived in terms of the derivatives of the director $\nabla_{i} n_{j}$ (see, for example, Refs. [1,26]). This method, however, has a number of complications as one has to take into consideration that $\mathbf{n}$ is a unit vector and that all expressions must be invariant under sign inversion of $\mathbf{n}$. The alternative approach employs the so called orientational deformation tensor $\gamma_{i j}=\epsilon_{i l k} n_{l} \nabla_{j} n_{k}$ where $\epsilon_{i j k}$ is the unitary antisymmetric Levi-Civita tensor.

The deformation tensor of the nematic phase can be derived, for example, in the following way. Let us consider an inhomogeneous nematic phase where $\mathbf{n}\left(\mathbf{r}_{1}\right)$ and $\mathbf{n}\left(\mathbf{r}_{2}\right)$ are the directors at the points $\mathbf{r}_{1}$ and $\mathbf{r}_{2}$, respectively. If the distance $r_{12}=\left|\mathbf{r}_{2}-\mathbf{r}_{1}\right|$ is sufficiently small, then the rotation of the director from the point $\mathbf{r}_{1}$ to the point $\mathbf{r}_{2}$ is characterized by the small angle $\delta \theta_{i}=\epsilon_{i j k} n_{j}\left(\mathbf{r}_{1}\right) n_{k}\left(\mathbf{r}_{2}\right)$. Performing the gradient expansion of $\mathbf{n}\left(\mathbf{r}_{2}\right)$ around $\mathbf{n}\left(\mathbf{r}_{1}\right)$

$$
\mathbf{n}\left(\mathbf{r}_{2}\right)=\mathbf{n}\left(\mathbf{r}_{1}\right)+\left(\mathbf{r}_{12} \cdot \nabla\right) \mathbf{n}\left(\mathbf{r}_{1}\right)+\ldots,
$$

one obtains

$$
\delta \theta_{i} \approx r_{12, j} \gamma_{i j}
$$

where only the terms linear in gradients are taken into account.
The free energy density of the distorted nematic phase can now be expanded in terms of $\gamma_{i j}$ keeping the quadratic terms:

$$
\mathcal{F}_{d}=\Lambda_{i j} \gamma_{i j}+K_{i j p q} \gamma_{i j} \gamma_{p q},
$$

where the material tensors $\Lambda_{i j}$ and $K_{i j p q}$ are defined in the homogeneous nematic state.

The first term in Eq. (2) is nonzero only in the chiral nematic phase. Indeed, $\gamma_{i j}$ is a pseudotensor while the freeenergy density $\mathcal{F}_{d}$ is a scalar and hence the material tensor $\Lambda_{i j}$ must also be a pseudotensor vanishing in the achiral nematic phase. Hence from the symmetry point of view, the tensor $\Lambda_{i j}$ can be expressed in the following general form:

$$
\Lambda_{i j}=\lambda_{0} \delta_{i j}+\lambda_{1} n_{i} n_{j}
$$

where $\lambda_{0}$ and $\lambda_{1}$ are pseudoscalars. Accordingly, the first term in Eq. (2) reduces to:

$$
\Lambda_{i j} \gamma_{i j}=\lambda_{0} \delta_{i j} \gamma_{i j}=-\lambda_{0}(\mathbf{n} \cdot(\boldsymbol{\nabla} \times \mathbf{n})) .
$$

This is the well-known chiral term in the distortion free energy which characterizes the helical twisting power in the chiral nematic phase.

The second term of Eq. (2) is invariant under the simultaneous interchange $i \rightarrow p$ and $j \rightarrow q$ and hence only the symmetric in those indexes part of the tensor $K_{i j p q}$ contributes to the elastic energy. Taking into account also the symmetry of the nematic phase, this part of the elasticity tensor can be expressed as:

$$
\begin{aligned}
K_{i j p q}= & \mathcal{K}_{0} \delta_{i p} \delta_{j q}+\mathcal{K}_{12}\left(\delta_{i j} \delta_{p q}+\delta_{i q} \delta_{j p}\right)+\mathcal{K}_{3} \delta_{i p} n_{j} n_{q} \\
& +\mathcal{K}_{4} \delta_{j q} n_{i} n_{p}+\mathcal{K}_{5}\left(\delta_{p q} n_{i} n_{j}+\delta_{i j} n_{p} n_{q}\right) \\
& +\mathcal{K}_{6}\left(\delta_{i q} n_{j} n_{p}+\delta_{j p} n_{i} n_{q}\right)+\mathcal{K}_{7} n_{i} n_{j} n_{p} n_{q} .
\end{aligned}
$$

One notes that the last four terms in Eq. (5) do not contribute to Eq. (2) as their contractions with $\gamma_{i j}$ or $\gamma_{p q}$ vanish since $n_{i} \gamma_{i j}=0$. The contraction of the term $\mathcal{K}_{12} \delta_{i j} \delta_{p q}$ with $\gamma_{i j} \gamma_{p q}$ yields the contribution proportional to the square of the twist deformation:

$$
\mathcal{K}_{12} \delta_{i j} \delta_{p q} \gamma_{i j} \gamma_{p q}=\mathcal{K}_{12}[\mathbf{n} \cdot(\nabla \times \mathbf{n})]^{2},
$$

and hence the parameter $\mathcal{K}_{12}$ contributes to the twist elastic constant $K_{22}$.

Contribution of other terms in Eq. (5) to the total distortion free energy can be evaluated using the local coordinate system in which the $z$ axis is parallel to the director $\mathbf{n}$ at the point r. In this coordinate system, $\nabla_{i} n_{z}=0$ for all $i$ because $\mathbf{n}$ is a unit vector. For the rest director derivatives one usually introduces convenient notations: $t_{1}=-\nabla_{x} n_{y}, t_{2}=\nabla_{y} n_{x}$, $s_{1}=\nabla_{x} n_{x}, s_{2}=\nabla_{y} n_{y}$, and $b_{1}=\nabla_{z} n_{x}, b_{2}=\nabla_{z} n_{y}$ (see, for example, Ref. [26]). Then the twist deformation can be expressed as

$$
[\mathbf{n} \cdot(\nabla \times \mathbf{n})]=-\left(t_{1}+t_{2}\right),
$$

while the splay and bend vectors read as:

$$
\mathbf{n}(\boldsymbol{\nabla} \cdot \mathbf{n})=\left(s_{1}+s_{2}\right) \mathbf{n},
$$

$$
(\mathbf{n} \cdot \nabla) \mathbf{n}=b_{1} \hat{\mathbf{x}}+b_{2} \hat{\mathbf{y}},
$$

correspondingly, where the unit vectors $\hat{\mathbf{x}}$ and $\hat{\mathbf{y}}$ are in the direction of the $x$ and $y$ axis of the local coordinate system, 
respectively. In the same local coordinate system, the components of orientational deformation tensor $\gamma_{i j}$ are expressed as $\gamma_{11}=t_{1}, \gamma_{12}=-s_{2}, \gamma_{13}=-b_{2}$ and $\gamma_{21}=s_{1}, \gamma_{22}=t_{2}, \gamma_{23}=$ $b_{1}$. The components $\gamma_{3 i}=0$ for all $i$.

Using these expressions one obtains:

$$
\begin{aligned}
\mathcal{K}_{0} \delta_{i p} \delta_{j q} \gamma_{i j} \gamma_{p q}= & \mathcal{K}_{0} \gamma_{i j} \gamma_{i j} \\
= & \mathcal{K}_{0}\left[\left(t_{1}+t_{2}\right)^{2}+\left(s_{1}+s_{2}\right)^{2}+b_{2}^{2}+b_{1}^{2}\right. \\
& \left.-2 t_{1} t_{2}-2 s_{1} s_{2}\right] \\
\mathcal{K}_{12} \delta_{i q} \delta_{j p} \gamma_{i j} \gamma_{p q}= & \mathcal{K}_{12}\left[\left(t_{1}+t_{2}\right)^{2}-2 t_{1} t_{2}-2 s_{1} s_{2}\right] \\
\mathcal{K}_{3} \delta_{i p} n_{j} n_{q} \gamma_{i j} \gamma_{p q}= & \mathcal{K}_{3}\left(b_{1}^{2}+b_{2}^{2}\right) .
\end{aligned}
$$

Using Eqs. (7)-(9) one can express $\left(s_{1}+s_{2}\right)^{2}=(\nabla$. $\mathbf{n})^{2}, \quad\left(t_{1}+t_{2}\right)^{2}=[\mathbf{n} \cdot(\nabla \times \mathbf{n})]^{2}$ and $b_{1}^{2}+b_{2}^{2}=[\mathbf{n} \times(\nabla \times$ n) $]^{2}$. The combination $-2\left(t_{1} t_{2}+s_{1} s_{2}\right)=\nabla \cdot[(\mathbf{n} \cdot \nabla) \mathbf{n}-$ $\mathbf{n}(\nabla \cdot \mathbf{n})]$ is a surface term which does not contain second derivatives of the director. As a result, the distortion freeenergy density (2) can be written in the familiar form:

$$
\begin{aligned}
\mathcal{F}_{d}= & -\lambda_{0}[\mathbf{n} \cdot(\nabla \times \mathbf{n})]+\frac{1}{2} K_{11}(\nabla \cdot \mathbf{n})^{2} \\
& +\frac{1}{2} K_{22}[\mathbf{n} \cdot(\nabla \times \mathbf{n})]^{2}+\frac{1}{2} K_{33}[\mathbf{n} \times(\nabla \times \mathbf{n})]^{2} \\
& +K_{s} \nabla \cdot[(\mathbf{n} \cdot \nabla) \mathbf{n}-\mathbf{n}(\nabla \cdot \mathbf{n})],
\end{aligned}
$$

where the Frank elastic constants can be expressed in terms of the coefficients in Eq. (5):

$$
\begin{gathered}
K_{11}=2 \mathcal{K}_{0}, \\
K_{22}=2\left(\mathcal{K}_{0}+2 \mathcal{K}_{12}\right), \\
K_{33}=2\left(\mathcal{K}_{0}+\mathcal{K}_{3}\right), \\
K_{s}=\mathcal{K}_{12}+\mathcal{K}_{0} .
\end{gathered}
$$

Thus the elastic constants of the nematic phase are expressed by three independent constants $\mathcal{K}_{0}, \mathcal{K}_{3}$, and $\mathcal{K}_{12}$ which enter the general expression for the elasticity tensor $\mathbf{K}$. One notes that the constant $\mathcal{K}_{12}$ determines the difference between $K_{11}$ and $K_{22}$ while the constant $\mathcal{K}_{3}$ specifies the difference between $K_{11}$ and $K_{33}$. Finally the Frank elastic constants can be expressed as the following contractions of the general elasticity tensor:

$$
\begin{aligned}
K_{11}= & \frac{3}{4} K_{i j p q}\left(\delta_{i p}-n_{i} n_{p}\right)\left(\delta_{j q}-n_{j} n_{q}\right) \\
& -\frac{1}{2} K_{i j p q}\left(\delta_{i q}-n_{i} n_{q}\right)\left(\delta_{j p}-n_{j} n_{p}\right), \\
K_{22}= & \frac{1}{4} K_{i j p q}\left(\delta_{i p}-n_{i} n_{p}\right)\left(\delta_{j q}-n_{j} n_{q}\right) \\
& +\frac{1}{2} K_{i j p q}\left(\delta_{i q}-n_{i} n_{q}\right)\left(\delta_{j p}-n_{j} n_{p}\right), \\
K_{33}= & K_{i j p q}\left(\delta_{i p}-n_{i} n_{p}\right) n_{j} n_{q} \\
& =K_{i j i q} n_{j} n_{q}-K_{i j p q} n_{i} n_{p} n_{j} n_{q},
\end{aligned}
$$

These general expressions relate conventional bulk elastic constants with the components of the elasticity tensor $\mathbf{K}$.
Particular expressions for the elasticity tensor can be obtained using a molecular filed theory of nematic LCs and are presented in the following section.

\section{MOLECULAR-STATISTICAL THEORY OF THE DISTORTED NEMATIC PHASE}

A molecular theory of elasticity of nematic LCs can be developed using the mean-field expression for the free energy of the inhomogeneous nematic phase. In this approximation (see, for example, Ref. [27]) the free energy is determined by the one-particle distribution function $f_{1}(\omega, \mathbf{r})$ which may also depend on position of the molecule $\mathbf{r}$ in the case of inhomogeneous director distribution $\mathbf{n}(\mathbf{r})$. Here the orientational degrees of freedom of a generally biaxial molecule are specified by $\omega=(\mathbf{a}, \mathbf{b})$, where the unit vectors $\mathbf{a}$ and $\mathbf{b}$ are in the directions of the long and short molecular axes, respectively.

One notes that in the system of polar molecules, the one particle distribution function can be approximately expressed as $[8,9,14] f_{1}(\omega, \mathbf{r})=f(\omega)[1+h(\omega, \mathbf{r})]$, where $f(\omega)$ is the orientational distribution function of the homogeneous nonpolar nematic phase and $h(\omega, \mathbf{r})$ is a small polar correction to the distribution function which exists only in the inhomogeneous state and is proportional to the gradients of the director. Such a polar correction to the distribution function eventually gives rise to the corresponding polar corrections to splay and bend elastic constants $[8,9,14]$. In this section, we consider the nematic phase composed of nonpolar molecules and hence $h(\omega, \mathbf{r})=0$. Then the distribution function $f$ is equal to that in the homogeneous nematic phase and may depend on the position $\mathbf{r}$ only via the inhomogeneous director $\mathbf{n}(\mathbf{r})$. The statistical theory of elasticity of polar nematic LCs is considered in Sec. VI.

We also assume for simplicity that the mesogenic molecules are effectively uniaxial (i.e., we neglect the ordering of short molecular axes for generally biaxial molecules [28]) and assume that the orientational distribution function and the corresponding pair interaction potential are independent of the short axes b. Hence in the uniaxial nematic LC $f_{1}(\omega, \mathbf{r})=f(\mathbf{a} \cdot \mathbf{n}(\mathbf{r}))$. The free energy in the molecular field approximation can then be expressed as

$$
\begin{aligned}
F= & \rho k_{B} T \int f[\mathbf{a} \cdot \mathbf{n}(\mathbf{r})] \ln [\Lambda f(\mathbf{a} \cdot \mathbf{n}(\mathbf{r}))] d \mathbf{a} d \mathbf{r} \\
& +\frac{1}{2} \rho^{2} \int V\left(\mathbf{a}_{1}, \mathbf{r}_{12}, \mathbf{a}_{2}\right) \\
& \times f\left[\mathbf{a}_{1} \cdot \mathbf{n}\left(\mathbf{r}_{1}\right)\right] f\left[\mathbf{a}_{2} \cdot \mathbf{n}\left(\mathbf{r}_{2}\right)\right] d \mathbf{r}_{12} d \mathbf{a}_{1} d \mathbf{a}_{2} d \mathbf{r}_{1},
\end{aligned}
$$

where $\Lambda$ is a constant, $\rho$ is the average molecular number density and $V\left(\mathbf{a}_{1}, \mathbf{r}_{12}, \mathbf{a}_{2}\right)$ is the effective pair intermolecular interaction potential which depends on the long axes $\mathbf{a}_{1}$ and $\mathbf{a}_{2}$ of the molecules 1 and 2 and on the intermolecular vector $\mathbf{r}_{12}=\mathbf{r}_{2}-\mathbf{r}_{1}$. the effective interaction potential may also include the steric cut-off factor which accounts for the fact that the moleculas cannot penetrate each other [29]. 
The effective interaction potential $V\left(\mathbf{a}_{1}, \mathbf{r}_{12}, \mathbf{a}_{2}\right)$ can be expanded in terms of the so-called spherical invariants [25,27]:

$$
V\left(\mathbf{a}_{1}, \mathbf{r}_{12}, \mathbf{a}_{2}\right)=\sum_{l m k} U_{l m k}\left(r_{12}\right) T^{l m k}\left(\mathbf{a}_{1}, \mathbf{u}_{12}, \mathbf{a}_{2}\right),
$$

where $\mathbf{u}_{12}=\mathbf{r}_{12} / r_{12}$ and the set $T^{l m k}\left(\mathbf{a}_{1}, \mathbf{u}_{12}, \mathbf{a}_{2}\right)$ is a complete set of basis functions that contain the vector $\mathbf{a}_{1}$ to the power $l$, the vector $\mathbf{u}_{12}$ to the power $m$ and the vector $\mathbf{a}_{2}$ to the power $k$. The explicit expressions for some invariants are given, for example, by van der Meer [24]. For convenience, we present the particular form of the relevant invariants $T^{l m k}$ and their key properties in Appendix A. The invariants with one zero index are just Legendre polynomials, i.e., $T^{n 0 n}\left(\mathbf{a}_{1}, \mathbf{u}_{12}, \mathbf{a}_{2}\right)=P_{n}\left(\mathbf{a}_{1} \cdot \mathbf{a}_{2}\right)$ where $P_{n}(x)$ is the Legendre polynomial of rank $n$.

The spherical invariants possess a number of important properties which simplify calculations. In particular the integral of $T^{l m k}\left(\mathbf{a}_{1}, \mathbf{u}_{12}, \mathbf{a}_{2}\right)$ with two arbitrary functions $g_{1}\left(\mathbf{a}_{1}\right.$. $\left.\mathbf{n}_{1}\right)$ and $g_{2}\left(\mathbf{a}_{2} \cdot \mathbf{n}_{2}\right)$ which depend only on $\mathbf{a}_{1}$ and $\mathbf{a}_{2}$ can be expressed as [24]:

$$
\begin{aligned}
& \int g_{1}\left(\mathbf{a}_{1} \cdot \mathbf{n}_{1}\right) T^{l m k}\left(\mathbf{a}_{1}, \mathbf{u}_{12}, \mathbf{a}_{2}\right) g_{2}\left(\mathbf{a}_{2} \cdot \mathbf{n}_{2}\right) d \mathbf{a}_{1} d \mathbf{a}_{2} \\
& =S_{1, l} S_{2, k} T^{l m k}\left(\mathbf{n}_{1}, \mathbf{u}_{12}, \mathbf{n}_{2}\right),
\end{aligned}
$$

where $S_{q, n}=\int d \mathbf{a} P_{n}(\mathbf{a} \cdot \mathbf{n}) g_{q}\left(\mathbf{a} \cdot \mathbf{n}_{q}\right)$ and where $q=1,2$. Equation (23) can also be used in the particular case when both $g_{1}(\mathbf{a} \cdot \mathbf{n})$ and $g_{2}(\mathbf{a} \cdot \mathbf{n})$ are equal to the orientational distribution function of the homogeneous nematic phase $f(\mathbf{a} \cdot \mathbf{n})$. In this case, $S_{1, l}$ and $S_{2, k}$ are the orientational nematic order parameters of rank $l$ and $k$, respectively.

Substituting the expansion Eq. (22) into the free-energy Eq. (21) and using the integration rule Eq. (23) one obtains:

$$
\begin{aligned}
F= & \rho k_{B} T \int f[\mathbf{a} \cdot \mathbf{n}(\mathbf{r})] \ln \{\Lambda f[\mathbf{a} \cdot \mathbf{n}(\mathbf{r})]\} d \mathbf{a} d \mathbf{r} \\
& +\frac{1}{2} \rho^{2} \sum_{l m k} S_{l} S_{k} \\
& \times \int U_{l m k}\left(r_{12}\right) T^{l m k}\left(\mathbf{n}\left(\mathbf{r}_{1}\right), \mathbf{u}_{12}, \mathbf{n}\left(\mathbf{r}_{2}\right)\right) d \mathbf{r}_{12} d \mathbf{r}_{1} .
\end{aligned}
$$

This free energy depends on the director at two different space points $\mathbf{r}_{1}$ and $\mathbf{r}_{2}$ and it is the free energy of the inhomogeneous nematic phase, which can be expressed as a sum of the free energy of the homogeneous state and the distortion free energy determined by the deformations of the director distribution. One notes that the first term in Eq. (24) is local and does not contribute to the distortion energy. For smoothly inhomogeneous director, the distortion free energy can be separated from the free energy of the homogeneous state by expanding the functions $T^{\text {lmk }}\left(\mathbf{n}\left(\mathbf{r}_{1}\right), \mathbf{u}_{12}, \mathbf{n}\left(\mathbf{r}_{2}\right)\right)$ in the second term of Eq. (24) in powers of $\mathbf{r}_{12}$ around $\mathbf{r}_{1}$.

Indeed, let us define $\tilde{T}^{l m k}\left(\mathbf{r}_{1}, \mathbf{u}_{12}, \mathbf{r}_{2}\right) \equiv T^{l m k}\left(\mathbf{n}\left(\mathbf{r}_{1}\right)\right.$, $\left.\mathbf{u}_{12}, \mathbf{n}\left(\mathbf{r}_{2}\right)\right)$ and expand them as functions of $\mathbf{r}_{2}$ in Taylor series around $\mathbf{r}_{1}$ :

$$
\tilde{T}^{l m k}\left(\mathbf{r}_{1}, \mathbf{u}_{12}, \mathbf{r}_{2}\right) \approx \tilde{T}^{l m k}\left(\mathbf{r}_{1}, \mathbf{u}_{12}, \mathbf{r}_{1}\right)+r_{12, j}\left[\frac{\partial}{\partial x_{2, j}} \tilde{T}^{l m k}\left(\mathbf{r}_{1}, \mathbf{u}_{12}, \mathbf{r}_{2}\right)\right]_{\mathbf{r}_{2}=\mathbf{r}_{1}}+\frac{r_{12, j} r_{12, q}}{2}\left[\frac{\partial^{2}}{\partial x_{2, j} \partial x_{2, q}} \tilde{T}^{l m k}\left(\mathbf{r}_{1}, \mathbf{u}_{12}, \mathbf{r}_{2}\right)\right]_{\mathbf{r}_{2}=\mathbf{r}_{1}}
$$

where the derivatives can be expressed as

$$
\left[\frac{\partial}{\partial x_{2, j}} \tilde{T}^{l m k}\left(\mathbf{r}_{1}, \mathbf{u}_{12}, \mathbf{r}_{2}\right)\right]_{\mathbf{r}_{2}=\mathbf{r}_{1}}=\frac{\partial n_{t}}{\partial x_{j}}\left(\mathbf{r}_{1}\right)\left[\frac{\partial}{\partial n_{2, t}} T^{l m k}\left(\mathbf{n}_{1}, \mathbf{u}_{12}, \mathbf{n}_{2}\right)\right]_{\mathbf{n}_{2}=\mathbf{n}_{1}=\mathbf{n}\left(\mathbf{r}_{1}\right)}
$$

and

$$
\begin{aligned}
{\left[\frac{\partial^{2}}{\partial x_{2, j} \partial x_{2, q}} \tilde{T}^{l m k}\left(\mathbf{r}_{1}, \mathbf{u}_{12}, \mathbf{r}_{2}\right)\right]_{\mathbf{r}_{2}=\mathbf{r}_{1}}=} & \frac{\partial^{2} n_{t}}{\partial x_{q} \partial x_{j}}\left(\mathbf{r}_{1}\right)\left[\frac{\partial}{\partial n_{2, t}} T^{l m k}\left(\mathbf{n}_{1}, \mathbf{u}_{12}, \mathbf{n}_{2}\right)\right]_{\mathbf{n}_{2}=\mathbf{n}_{1}=\mathbf{n}\left(\mathbf{r}_{1}\right)} \\
& +\frac{\partial n_{t}}{\partial x_{j}}\left(\mathbf{r}_{1}\right) \frac{\partial n_{s}}{\partial x_{q}}\left(\mathbf{r}_{1}\right)\left[\frac{\partial^{2} T^{l m k}}{\partial n_{2, t} \partial n_{2, s}}\left(\mathbf{n}_{1}, \mathbf{u}_{12}, \mathbf{n}_{2}\right)\right]_{\mathbf{n}_{2}=\mathbf{n}_{1}=\mathbf{n}\left(\mathbf{r}_{1}\right)}
\end{aligned}
$$

As the integral of the term linear in $r_{12, j}$ in Eq. (25) vanishes identically for achiral molecules (i.e., for any centrosymmetric interaction potential) the free-energy Eq. (24) naturally splits into the energy of homogeneous nematic state:

$$
\begin{aligned}
F_{h}= & \rho k_{B} T V \int f(\mathbf{a} \cdot \mathbf{n}) \ln \left[\Lambda f(\mathbf{a} \cdot \mathbf{n}] d \mathbf{a}+\frac{1}{2} \rho^{2} V \sum_{l m k} \int d \mathbf{r}_{12} U_{l m k}\left(r_{12}\right)\right. \\
& \times \int f\left(\mathbf{a}_{1} \cdot \mathbf{n}\right) T^{l m k}\left(\mathbf{a}_{1}, \mathbf{u}_{12}, \mathbf{a}_{2}\right) f\left(\mathbf{a}_{2} \cdot \mathbf{n}\right) d \mathbf{a}_{1} d \mathbf{a}_{2}
\end{aligned}
$$

and the part describing the distortion free energy:

$$
F_{d}=\frac{1}{4} \rho^{2} \sum_{l m k} S_{l} S_{k} \int d \mathbf{r}_{12} r_{12, j} r_{12, q} U_{l m k}\left(r_{12}\right) \int d \mathbf{r}_{1}\left[\frac{\partial^{2}}{\partial x_{2, j} \partial x_{2, q}} \tilde{T}^{l m k}\left(\mathbf{r}_{1}, \mathbf{u}_{12}, \mathbf{r}_{2}\right)\right]_{\mathbf{r}_{2}=\mathbf{r}_{1}} .
$$


On substituting Eq. (27) into $F_{d}$ one notices that the integral over $\mathbf{r}_{1}$ of the first term of Eq. (27) containing the second derivative of the director can be taken by parts as:

$$
\begin{aligned}
& \int d \mathbf{r}_{1} \frac{\partial^{2} n_{t}}{\partial x_{q} \partial x_{j}}\left(\mathbf{r}_{1}\right)\left[\frac{\partial}{\partial n_{2, t}} T^{l m k}\left(\mathbf{n}_{1}, \mathbf{u}_{12}, \mathbf{n}_{2}\right)\right]_{\mathbf{n}_{2}=\mathbf{n}_{1}=\mathbf{n}\left(\mathbf{r}_{1}\right)} \\
& =-\int d \mathbf{r}_{1} \frac{\partial n_{t}}{\partial x_{j}}\left(\mathbf{r}_{1}\right) \frac{\partial n_{s}}{\partial x_{q}}\left(\mathbf{r}_{1}\right)\left[\frac{\partial^{2} T^{l m k}}{\partial n_{2, t} \partial n_{1, s}}\left(\mathbf{n}_{1}, \mathbf{u}_{12}, \mathbf{n}_{2}\right)+\frac{\partial^{2} T^{l m k}}{\partial n_{2, t} \partial n_{2, s}}\left(\mathbf{n}_{1}, \mathbf{u}_{12}, \mathbf{n}_{2}\right)\right]_{\mathbf{n}_{2}=\mathbf{n}_{1}=\mathbf{n}\left(\mathbf{r}_{1}\right)},
\end{aligned}
$$

and the second term here obviously cancels out the integral over $\mathbf{r}_{1}$ of the second term in Eq. (27). Therefore, one obtains the distortion free energy (29) as:

$$
F_{d}=-\frac{1}{4} \rho^{2} \sum_{l m k} S_{l} S_{k} \int d \mathbf{r}_{12} r_{12, j} r_{12, q} U_{l m k}\left(r_{12}\right) \int d \mathbf{r}_{1} \frac{\partial n_{t}}{\partial x_{j}}\left(\mathbf{r}_{1}\right) \frac{\partial n_{s}}{\partial x_{q}}\left(\mathbf{r}_{1}\right)\left[\frac{\partial^{2} T^{l m k}}{\partial n_{2, t} \partial n_{1, s}}\left(\mathbf{n}_{1}, \mathbf{u}_{12}, \mathbf{n}_{2}\right)\right]_{\mathbf{n}_{2}=\mathbf{n}_{1}=\mathbf{n}\left(\mathbf{r}_{1}\right)} .
$$

Next, one notices that

$$
\frac{\partial n_{t}}{\partial x_{j}}=-\epsilon_{t v i} n_{v} \gamma_{i j}
$$

Substituting the director derivatives in this form in Eq. (31), one obtains:

$$
F_{d}=-\frac{1}{4} \rho^{2} \sum_{l m k} S_{l} S_{k} \int d \mathbf{r}_{12} r_{12, j} r_{12, q} U_{l m k}\left(r_{12}\right) \int d \mathbf{r}_{1} \epsilon_{t v i} n_{v} \gamma_{i j} \epsilon_{s u p} n_{u} \gamma_{p q}\left[\frac{\partial^{2} T^{l m k}}{\partial n_{2, t} \partial n_{1, s}}\left(\mathbf{n}_{1}, \mathbf{u}_{12}, \mathbf{n}_{2}\right)\right]_{\mathbf{n}_{2}=\mathbf{n}_{1}=\mathbf{n}\left(\mathbf{r}_{1}\right)},
$$

which reduces the distortion energy density to the general form (2) and enables one to express the energy as

$$
F_{d}=\sum_{l m k} \int d \mathbf{r} K_{i j p q}^{l m k} \gamma_{i j} \gamma_{p q}
$$

and the elasticity tensor is reduced to a sum $K_{i j p q}=\sum_{l m k} K_{i j p q}^{l m k}$ of different contributions expressed as:

$$
K_{i j p q}^{l m k}=-\frac{1}{4} \rho^{2} S_{l} S_{k} \epsilon_{t v i} \epsilon_{s u p} n_{v} n_{u} \int d \mathbf{r} r_{j} r_{q} U_{l m k}(r)\left[\frac{\partial^{2} T^{l m k}}{\partial n_{2, t} \partial n_{1, s}}\left(\mathbf{n}_{1}, \frac{\mathbf{r}}{r}, \mathbf{n}_{2}\right)\right]_{\mathbf{n}_{2}=\mathbf{n}_{1}=\mathbf{n}} .
$$

The relationship between the elasticity tensor (29) and (35) and the general results obtained by Poniewierski and Stechi [22] is discussed in Appendix B.

\section{ORDER PARAMETERS AND FRANK ELASTIC CONSTANTS OF NONPOLAR NEMATICS}

In practice, the series in Eq. (22) should be truncated to take into account the first few relevant terms. Here we preserve all nonpolar invariants up to the fourth order in $\mathbf{a}_{1}$ and $\mathbf{a}_{2}$ and up to the quadratic order in $\mathbf{u}_{12}$. Then the effective interaction potential is approximately expressed as:

$$
\begin{aligned}
& V\left(\mathbf{a}_{1}, \mathbf{r}_{12}, \mathbf{a}_{2}\right) \approx U_{0}+U_{202}\left(r_{12}\right) T^{202}\left(\mathbf{a}_{1}, \mathbf{u}_{12}, \mathbf{a}_{2}\right) \\
& \quad+U_{220}\left(r_{12}\right) T^{220}\left(\mathbf{a}_{1}, \mathbf{u}_{12}, \mathbf{a}_{2}\right)+U_{022}\left(r_{12}\right) T^{022}\left(\mathbf{a}_{1}, \mathbf{u}_{12}, \mathbf{a}_{2}\right) \\
& \quad+U_{222}\left(r_{12}\right) T^{222}\left(\mathbf{a}_{1}, \mathbf{u}_{12}, \mathbf{a}_{2}\right)+U_{422}\left(r_{12}\right) T^{422}\left(\mathbf{a}_{1}, \mathbf{u}_{12}, \mathbf{a}_{2}\right) \\
& \quad+U_{224}\left(r_{12}\right) T^{224}\left(\mathbf{a}_{1}, \mathbf{u}_{12}, \mathbf{a}_{2}\right)+U_{404}\left(r_{12}\right) T^{404}\left(\mathbf{a}_{1}, \mathbf{u}_{12}, \mathbf{a}_{2}\right)
\end{aligned}
$$

\section{A. Orientational order parameters}

If the orientational deformations in the nematic phase are smooth on the molecular interaction scale, then one may neglect their effect on the nematic order parameters and evaluate them by minimizing the free energy of the homogeneous phase given by Eq. (28). Varying the free energy as a functional of $F_{h}[f(\mathbf{a} \cdot \mathbf{n})]$ one obtains the integral equation:

$$
f\left(\mathbf{a}_{1} \cdot \mathbf{n}\right)=Z^{-1} \exp \left[-U_{\mathrm{MF}}\left(\mathbf{a}_{1} \cdot \mathbf{n}\right) / k_{B} T\right]
$$

where $Z$ is the normalization factor and $U_{\mathrm{MF}}\left(\mathbf{a}_{1} \cdot \mathbf{n}\right)$ is the mean-field potential which is expressed as:

$$
U_{\mathrm{MF}}\left(\mathbf{a}_{1} \cdot \mathbf{n}\right)=\rho \sum_{l m k} S_{k} \int d \mathbf{r}_{12} U_{l m k}\left(r_{12}\right) T^{l m k}\left(\mathbf{a}_{1}, \mathbf{u}_{12}, \mathbf{n}\right)
$$

where we have taken into account that in the homogeneous nematic phase $\mathbf{n}\left(\mathbf{r}_{1}\right)=\mathbf{n}\left(\mathbf{r}_{2}\right)=\mathbf{n}=$ const.

Integrating over $\mathbf{r}_{12}$ and using the fact that for $m>0$, $\int T^{l m k}\left(\mathbf{a}, \mathbf{u}_{12}, \mathbf{n}\right) d^{2} \mathbf{u}_{12}=0$, together with Eq. (23), one finally obtains the following approximate expression for the mean-field potential where all terms with $k, l, m \leqslant 4$ in the expansion (36) are taken into account:

$$
U_{\mathrm{MF}}\left(\mathbf{a}_{1} \cdot \mathbf{n}\right)=\rho S \mathcal{V}^{202} P_{2}\left(\mathbf{a}_{1} \cdot \mathbf{n}\right)+\rho S_{4} \mathcal{V}^{404} P_{4}\left(\mathbf{a}_{1} \cdot \mathbf{n}\right) .
$$

Here we have omitted the index of the order parameter $S_{2}$ to follow the historical notations, and introduced the parameters $\mathcal{V}^{\alpha 0 \alpha}$ according to the general rule (A17).

Substituting Eq. (39) into Eqs. (37) and (28) one obtains the final expression for the homogeneous nematic free-energy 
density:

$$
\begin{aligned}
\frac{1}{V} F_{h}= & -\frac{1}{2} \rho^{2} S^{2} \mathcal{V}^{202}-\frac{1}{2} \rho^{2} S_{4}^{2} \mathcal{V}^{404} \\
& -\rho k_{B} T \ln \int \exp \left[-U_{\mathrm{MF}}(\mathbf{a} \cdot \mathbf{n}) / k_{B} T\right] d \mathbf{a},
\end{aligned}
$$

where $U_{\mathrm{MF}}(\mathbf{a} \cdot \mathbf{n})$ is given by Eq. (39). This free energy is fully determined by the two order parameters, $S$ and $S_{4}$, which can be obtained by its numerical minimization.

\section{B. Frank elastic constants}

As shown in Appendix C, substitution of the effective pair interaction potential (36) into Eq. (35) allows obtaining the elastic constants (18-20) as:

$$
\begin{aligned}
K_{11}= & 2 \pi \rho^{2} S\left(-S \mathcal{U}^{202}-\frac{1}{5} S \mathcal{U}^{222}+S_{4} \mathcal{U}^{422}\right) \\
& -\frac{20}{3} \pi \rho^{2} S_{4}^{2} \mathcal{U}^{404}, \\
K_{22}= & 2 \pi \rho^{2} S\left(-S \mathcal{U}^{202}+\frac{2}{5} S \mathcal{U}^{222}+\frac{1}{3} S_{4} \mathcal{U}^{422}\right) \\
& -\frac{20}{3} \pi \rho^{2} S_{4}^{2} \mathcal{U}^{404}, \\
K_{33}= & 2 \pi \rho^{2} S\left(-S \mathcal{U}^{202}-\frac{1}{5} S \mathcal{U}^{222}-\frac{4}{3} S_{4} \mathcal{U}^{422}\right) \\
& -\frac{20}{3} \pi \rho^{2} S_{4}^{2} \mathcal{U}^{404},
\end{aligned}
$$

where all constants $\mathcal{U}^{l m k}$ are evaluated according to the general rule (A18).

\section{ORDER PARAMETERS AND ELASTIC CONSTANTS FOR MOLECULES INTERACTING VIA THE GAY-BERNE POTENTIAL}

As the elastic constants expressed by Eqs. (18)-(20) explicitly depend on two order parameters, $S$ and $S_{4}$, their evaluation requires a particular model, relying on a specific molecular interaction potential. Typically in LCs, the main part of the molecular interaction potential is determined by the long-range attraction and short-range repulsion of hard elongated molecules.

\section{A. Gay-Berne pair interaction potential}

A useful model potential of that kind is the G-B potential [30] which combines relative simplicity, continuity, and capability of reproducing orientational order of anisotropic liquids $[31,32]$. It expresses the pair intermolecular interaction energy as an anisotropic generalization of the Lenard-Jones potential:

$$
\begin{aligned}
U_{\mathrm{GB}}\left(\mathbf{a}_{1}, \mathbf{r}_{12}, \mathbf{a}_{2}\right)= & 4 \varepsilon\left(\mathbf{a}_{1}, \mathbf{u}, \mathbf{a}_{2}\right) \\
& \times\left\{\left[r_{12} / r_{0}-\sigma\left(\mathbf{a}_{1}, \mathbf{u}, \mathbf{a}_{2}\right)+1\right]^{-12}\right. \\
& \left.-\left[r_{12} / r_{0}-\sigma\left(\mathbf{a}_{1}, \mathbf{u}, \mathbf{a}_{2}\right)+1\right]^{-6}\right\}
\end{aligned}
$$

with the range

$$
\begin{aligned}
& \sigma\left(\mathbf{a}_{1}, \mathbf{u}, \mathbf{a}_{2}\right) \\
& \quad=\left\{1-\frac{\chi}{2}\left[\frac{\left(\mathbf{u} \cdot \mathbf{a}_{1}+\mathbf{u} \cdot \mathbf{a}_{2}\right)^{2}}{1+\chi \mathbf{a}_{1} \cdot \mathbf{a}_{2}}+\frac{\left(\mathbf{u} \cdot \mathbf{a}_{1}-\mathbf{u} \cdot \mathbf{a}_{2}\right)^{2}}{1-\chi \mathbf{a}_{1} \cdot \mathbf{a}_{2}}\right]\right\}^{-1 / 2}
\end{aligned}
$$

and strength

$$
\begin{aligned}
\varepsilon\left(\mathbf{a}_{1},\right. & \left.\mathbf{u}, \mathbf{a}_{2}\right) \\
= & \varepsilon_{0}\left[1-\chi^{2}\left(\mathbf{a}_{1} \cdot \mathbf{a}_{2}\right)^{2}\right]^{-v / 2} \\
& \times\left\{1-\frac{\chi^{\prime}}{2}\left[\frac{\left(\mathbf{u} \cdot \mathbf{a}_{1}+\mathbf{u} \cdot \mathbf{a}_{2}\right)^{2}}{1+\chi^{\prime} \mathbf{a}_{1} \cdot \mathbf{a}_{2}}+\frac{\left(\mathbf{u} \cdot \mathbf{a}_{1}-\mathbf{u} \cdot \mathbf{a}_{2}\right)^{2}}{1-\chi^{\prime} \mathbf{a}_{1} \cdot \mathbf{a}_{2}}\right]\right\}^{\mu} .
\end{aligned}
$$

depending on the orientations of long molecular axes.

Two parameters characterize the effective molecular shape: $r_{0}$ is its breadth and $\chi=\left(\kappa^{2}-1\right) /\left(\kappa^{2}+1\right)$ is determined by the relative molecule elongation $\kappa$. The parameter $\chi^{\prime}=$ $\left(\kappa^{\prime 1 / \mu}-1\right) /\left(\kappa^{\prime 1 / \mu}+1\right)$ is determined by the ratio $\kappa^{\prime}$ of the well depths for side-to-side and end-to-end molecular orientations.

The parameters $\mu$ and $v$ enumerate certain known modifications of the potential. Thus, the values $\mu=2, v=1$ correspond to the potential originally proposed in Ref. [30], while other sets, such as $\mu=1, v=2$, and $\mu=1, v=3$, have been studied later in Refs. [33] and [32,34] correspondingly.

One notes that in the generalized mean-field approximation used in this paper the G-B potential (44) is to be multiplied by a step function $\Theta\left(r_{12}-r_{0} \sigma\left(\mathbf{a}_{1}, \mathbf{u}, \mathbf{a}_{2}\right)\right)$ which excludes from the integration in Eq. (38) the anisometric volume $r_{12}<$ $r_{0} \sigma\left(\mathbf{a}_{1}, \mathbf{u}, \mathbf{a}_{2}\right)$ where the potential is positive. This steric cutoff factor removes the contribution of certain intermolecular configurations when the interacting anisotropic molecules are too close to each other and effectively intersect.

\section{B. Expansion coefficients}

The analytical dependence of G-B potential on the intermolecular distance $r_{12}$ allows expressing the coefficients (A17) as

$$
\begin{aligned}
\mathcal{V}^{\alpha 0 \alpha}= & \frac{-r_{0}^{3}}{\left\|T^{\alpha 0 \alpha}\right\|} \int d \mathbf{a}_{1} d \mathbf{a}_{2} T^{\alpha 0 \alpha}\left(\mathbf{a}_{1}, \mathbf{u}, \mathbf{a}_{2}\right) \\
& \times \frac{2 \varepsilon\left(\mathbf{a}_{1}, \mathbf{u}, \mathbf{a}_{2}\right)}{495}\left[31+81 \sigma\left(\mathbf{a}_{1}, \mathbf{u}, \mathbf{a}_{2}\right)\right. \\
& \left.+108 \sigma^{2}\left(\mathbf{a}_{1}, \mathbf{u}, \mathbf{a}_{2}\right)\right],
\end{aligned}
$$

while the coefficients (A18) reduce to

$$
\begin{aligned}
\mathcal{U}^{l m k}= & \frac{-r_{0}^{5}}{\left\|T^{l m k}\right\|} \int d \mathbf{a}_{1} d \mathbf{a}_{2} T^{l m k}\left(\mathbf{a}_{1}, \mathbf{u}, \mathbf{a}_{2}\right) \\
& \times \frac{2 \varepsilon\left(\mathbf{a}_{1}, \mathbf{u}, \mathbf{a}_{2}\right)}{1155}\left[461+455 \sigma\left(\mathbf{a}_{1}, \mathbf{u}, \mathbf{a}_{2}\right)\right. \\
& +434 \sigma^{2}\left(\mathbf{a}_{1}, \mathbf{u}, \mathbf{a}_{2}\right)+378 \sigma^{3}\left(\mathbf{a}_{1}, \mathbf{u}, \mathbf{a}_{2}\right) \\
& \left.+252 \sigma^{4}\left(\mathbf{a}_{1}, \mathbf{u}, \mathbf{a}_{2}\right)\right] .
\end{aligned}
$$

Numerically evaluating the remaining integrals we obtain the dimensionless parameters $v^{l m k}=\mathcal{V}^{l m k}\left(\varepsilon_{0} r_{0}^{3}\right)^{-1}$ and $u^{l m k}=$ $\mathcal{U}^{l m k}\left(\varepsilon_{0} r_{0}^{5}\right)^{-1}$ that determine the phase behavior and elastic constants. Those corresponding to the classical G-B potential with $\mu=2, v=1$ are presented in Fig. 1, while the constants of modified potentials with $\mu=1, v=2$ and with $\mu=1$, $v=3$ are shown in Figs. 2 and 3, respectively. As discussed in Appendix D, the moderate values of the parameters of 

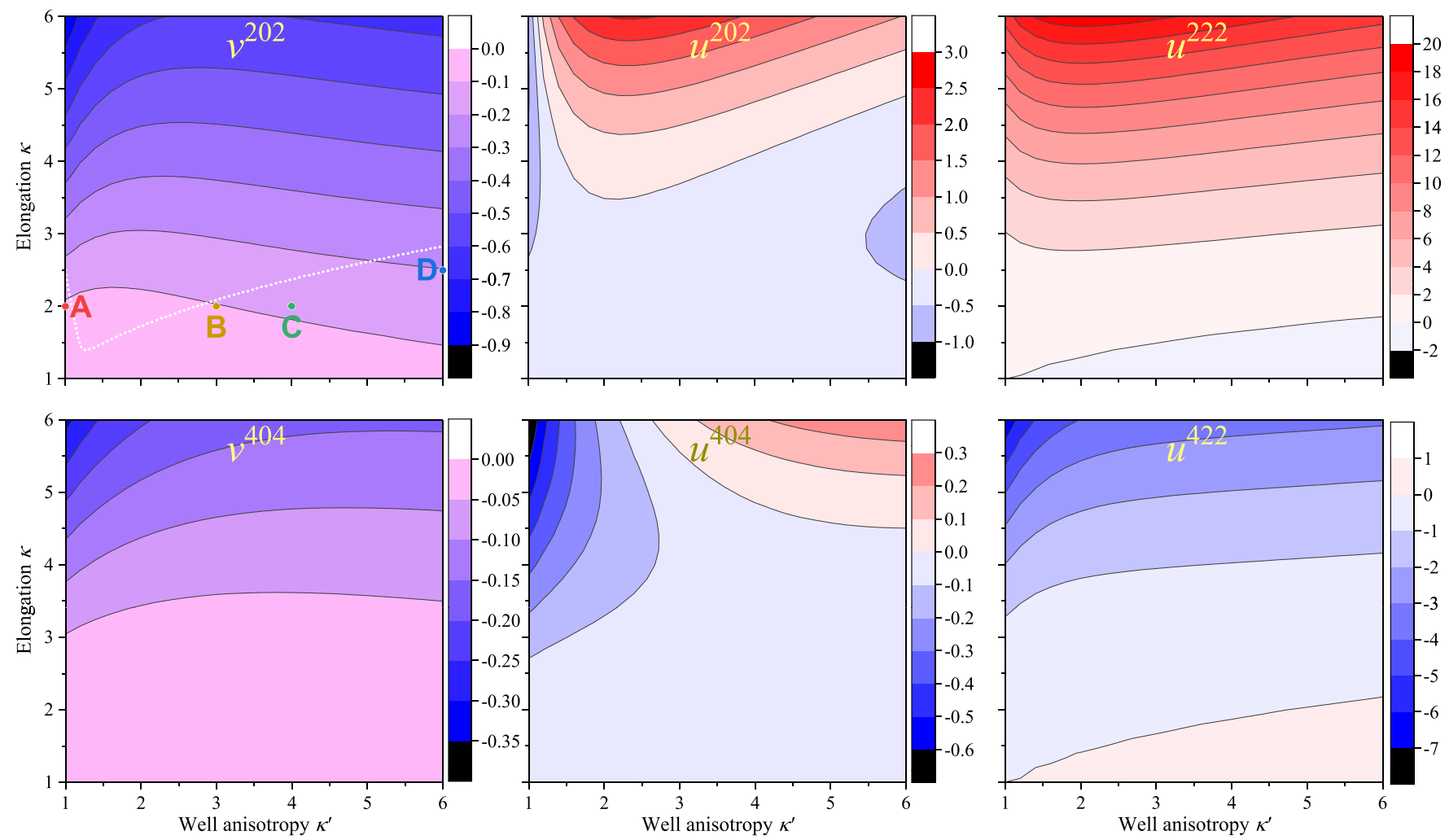

FIG. 1. Colormaps of the dimensionless parameters of the classical G-B pair potential with $\mu=2$ and $v=1$ characterizing its expansion in spherical invariants determining the nematic ordering $\left(v^{202}\right.$ and $\left.v^{404}\right)$ and the elastic constants $\left(u^{202}, u^{404}, u^{222}\right.$, and $\left.u^{422}\right)$. The white dotted line of the border between the areas of positive and negative elastic constants and four exemplary points A, B, C, and D are shown in the $v^{202}$ plot.
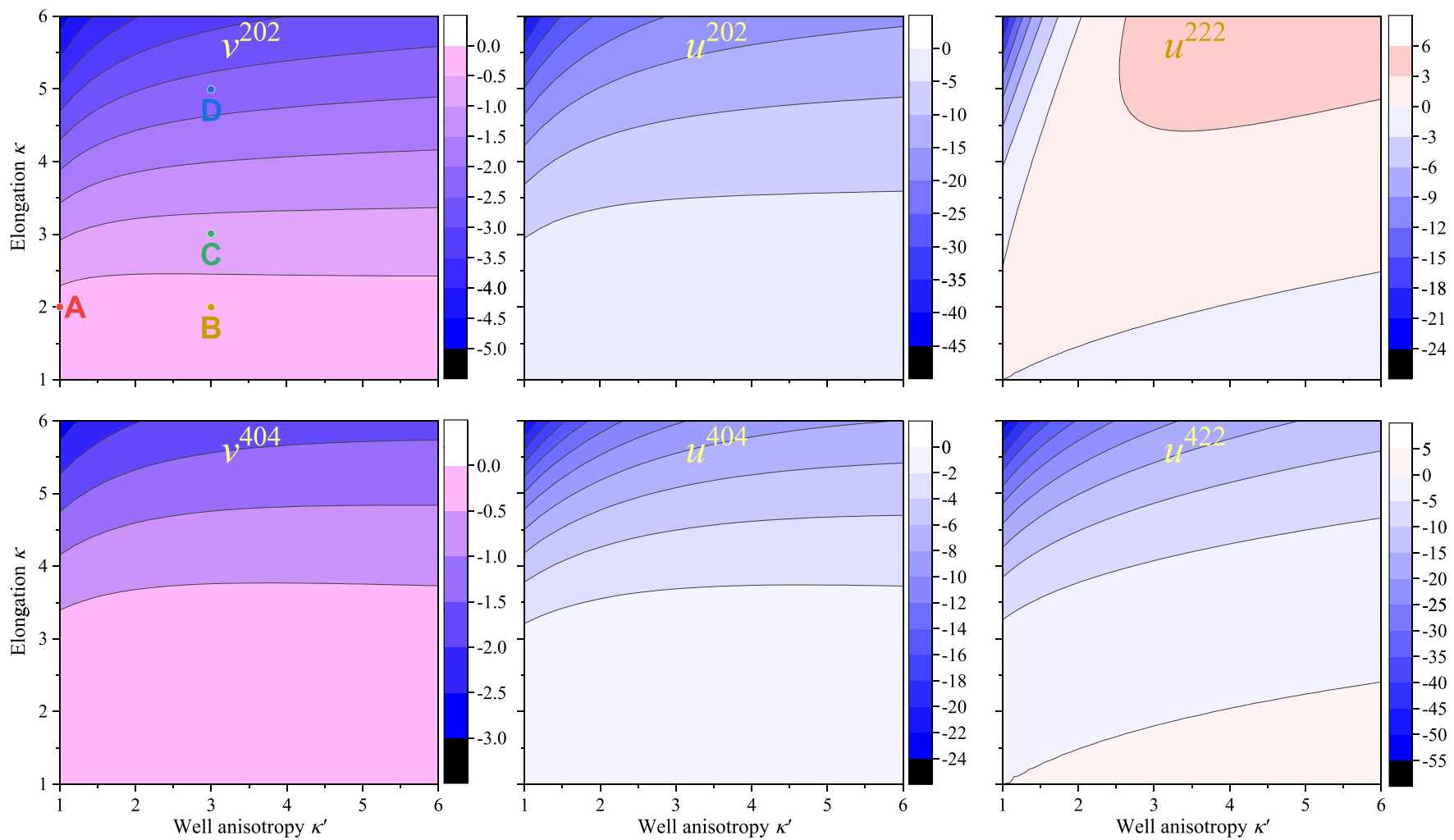

FIG. 2. Colormaps of the dimensionless parameters of the modified G-B pair potential with $\mu=1$ and $v=2$ characterizing its expansion in spherical invariants determining the nematic ordering $\left(v^{202}\right.$ and $\left.v^{404}\right)$ and elastic constants $\left(u^{202}, u^{404}, u^{222}\right.$, and $\left.u^{422}\right)$. Four exemplary points $\mathrm{A}, \mathrm{B}, \mathrm{C}$, and $\mathrm{D}$ are marked in the $v^{202}$ plot. 

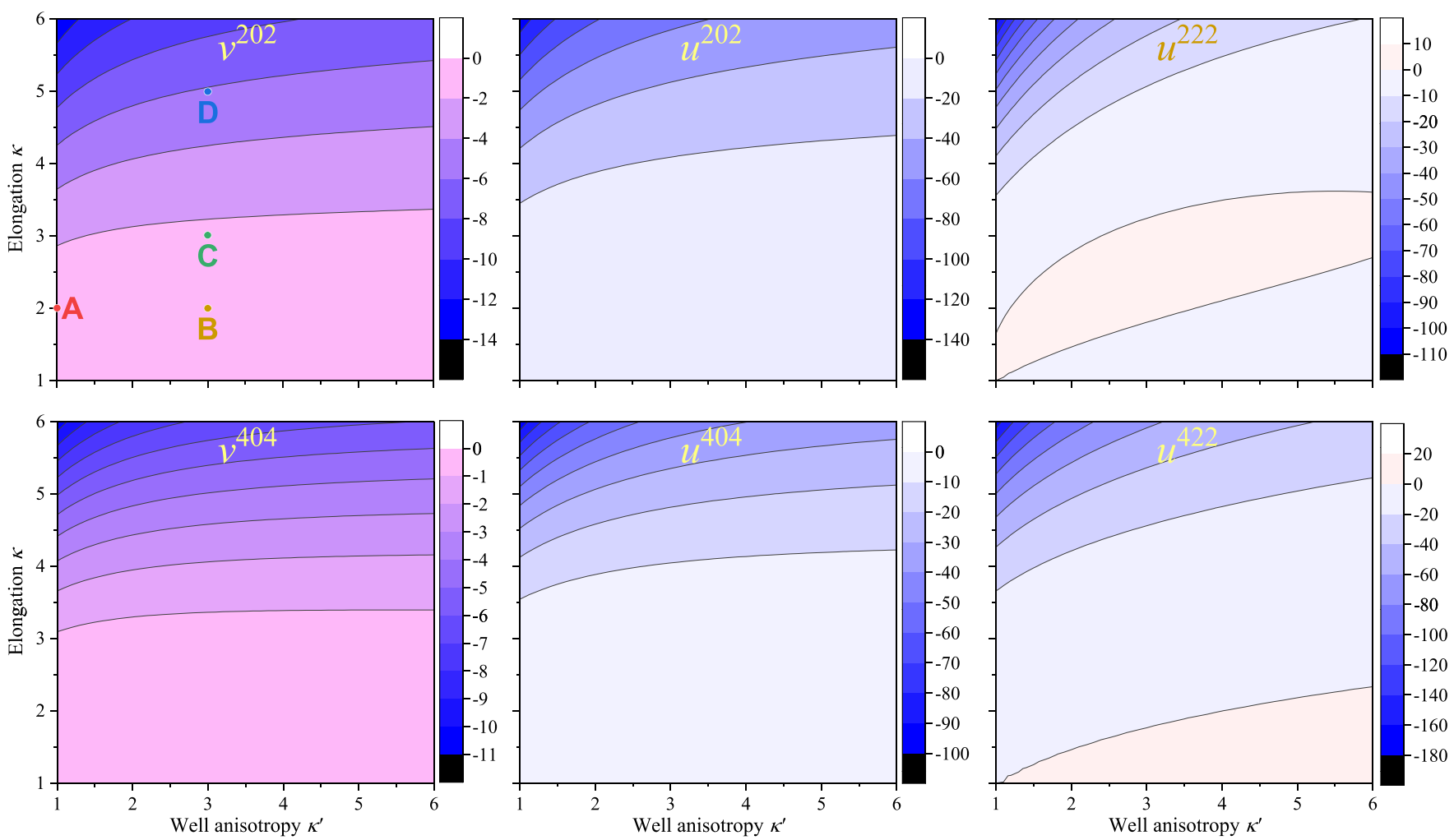

FIG. 3. Same as Fig. 2 for the modified G-B pair potential with $\mu=1$ and $v=3$.

the classical G-B potential arise as a result of substantial compensation of much larger contributions. For the modified $\mathrm{G}-\mathrm{B}$ potential, this compensation is less pronounced and the dimensionless constants of this potential are all negative and by one-two orders of magnitude larger at large $\kappa$.

\section{Elastic constants of the Gay-Berne nematic}

Using the evaluated dimensionless parameters of the potentials we calculate the order parameters $S$ and $S_{4}$ by a direct minimization of the free energy (40) and obtain them as functions of the dimensionless temperature $\tau=$ $k_{B} T\left(\left|v^{202}\right| \varepsilon_{0} \rho r_{0}^{3}\right)^{-1}$. Note that the latter includes the absolute value of the parameter $v^{202}$, which allows us to rescale the temperature dependencies into very similar ranges.

The elastic constants are determined by the order parameters and the coefficients $\mathcal{U}^{l m k}$ according to Eqs. (41)-(43). We express them by the dimensionless constants $k_{i i}=$ $K_{i i}\left(\varepsilon_{0} \rho^{2} r_{0}^{5}\right)^{-1}$ determined by the dimensionless parameters $u^{l m k}$ :

$$
\begin{aligned}
& k_{11}=-2 \pi S^{2} u^{202}-\frac{2}{5} \pi S^{2} u^{222}+2 \pi S S_{4} u^{422}-\frac{20}{3} \pi S_{4}^{2} u^{404}, \\
& k_{22}=-2 \pi S^{2} u^{202}+\frac{4}{5} \pi S^{2} u^{222}+\frac{2}{3} \pi S S_{4} u^{422}-\frac{20}{3} \pi S_{4}^{2} u^{404}, \\
& k_{33}=-2 \pi S^{2} u^{202}-\frac{2}{5} \pi S^{2} u^{222}-\frac{8}{3} \pi S S_{4} u^{422}-\frac{20}{3} \pi S_{4}^{2} u^{404} .
\end{aligned}
$$

The stability of nematic phase naturally requires all elastic constants to be positive. As shown in Fig. 1, this substantially reduces the appropriate range of $\kappa$ for the original G-B po- tential. The main factor is the large parameter $u^{222}$, which negatively contributes to the elastic constants when $\kappa$ exceeds 2. As shown in Appendix D, several vector combinations entering $u^{222}$ with different signs are all very large, but they substantially compensate each other yielding moderate values of $u^{222}$.

Within the range of positive elastic constants, we choose four well-separated points marked as A, B, C, and D in Fig. 1 and present the corresponding temperature variations of the order parameters in Fig. 4(a) and the dimensionless elastic constants in Fig. 4(b). One can readily see that the temperature variation of the order parameters is very similar for all four cases. At the same time, the variation of the elastic constants is very different: The difference between the constants can be rather small (as for the point B) or sufficiently large. One notes, however, that in all cases $K_{22}$ is the largest elastic constant which is in contradiction with typical experimental data for nematic LCs consisting of prolate molecules. This is another disadvantage of the classical G-B potential.

For the modified G-B potentials with $\mu=1, v=2$ and $\mu=1, \nu=3$ the whole range of the parameters $\kappa$ and $\kappa^{\prime}$ corresponds to a stable nematic phase with positive elastic constants. For four well separated points marked as A, B, C, and $\mathrm{D}$ in Figs. 2 and 3, we present the corresponding temperature variation of the order parameters and dimensionless elastic constants in Figs. 5 and 6, respectively. One notes that the points $\mathrm{B}, \mathrm{C}$, and $\mathrm{D}$ correspond to the same value of the parameter $\kappa$. Comparing the graphs B, C, and D in Figs, 5 and 6 one concludes that the relative difference between the elastic constants is increasing with the increasing molecular elongation. Comparing also the graphs $\mathrm{A}$ and $\mathrm{B}$, one may 


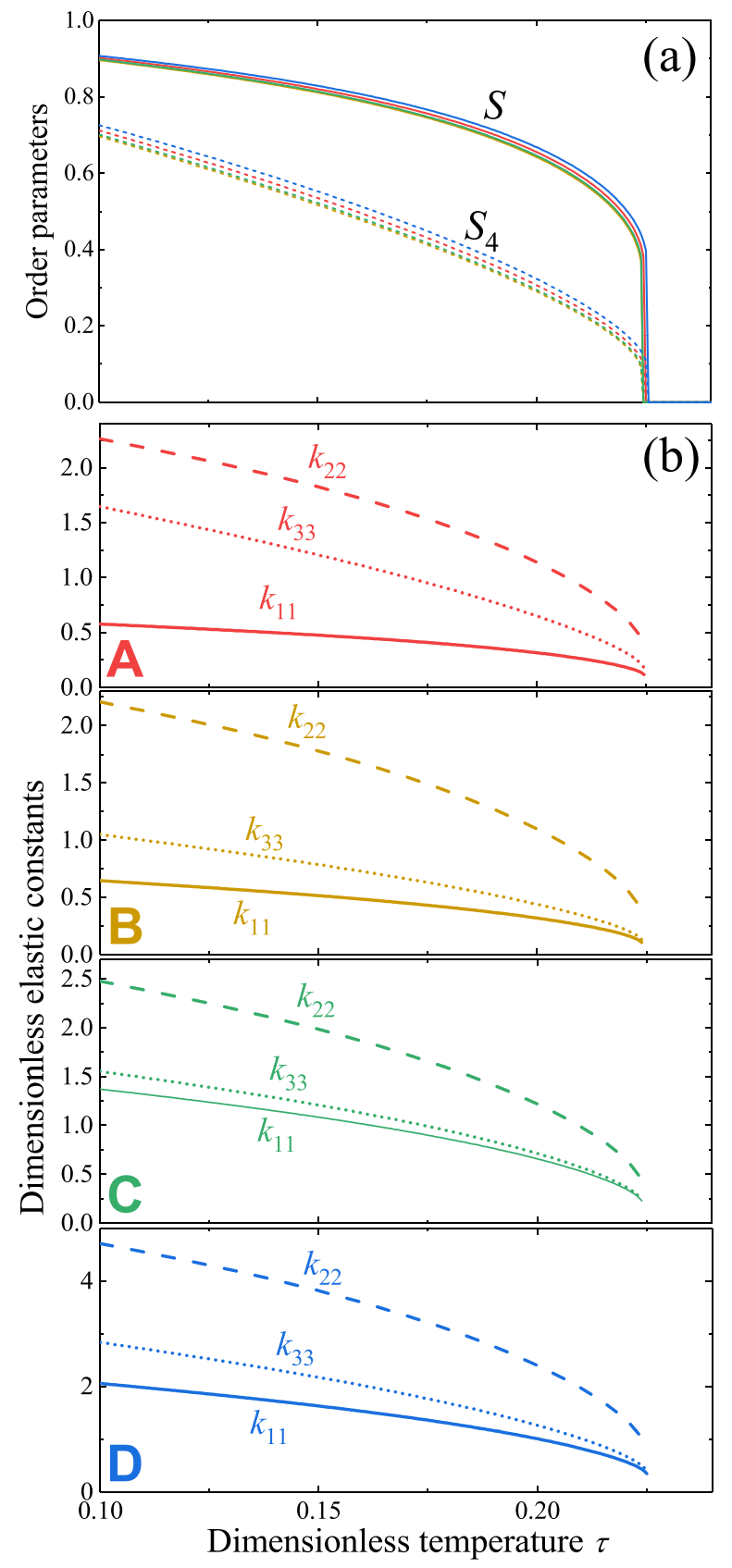

FIG. 4. Thermodynamics of nematic liquid crystal determined by the parameters of classical G-B potential with $\mu=2, v=1$ (see Fig. 1): (a) Dependencies of the order parameters $S$ and $S_{4}$ and (b) dimensionless elastic constants $k_{11}$ (solid), $k_{22}$ (dashed), and $k_{33}$ (dotted) on the dimensionless temperature for four sets of parameters corresponding to the points A, B, C, and D,marked in Fig. 1. Same colors correspond to same points.

assume that this difference also decreases with the increasing anisotropy of the potential well. Note also that the points A and $B$ are the same for all three G-B potentials and one can see that the elastic constants of the classical G-B potential are significantly different from those for the modified potentials.

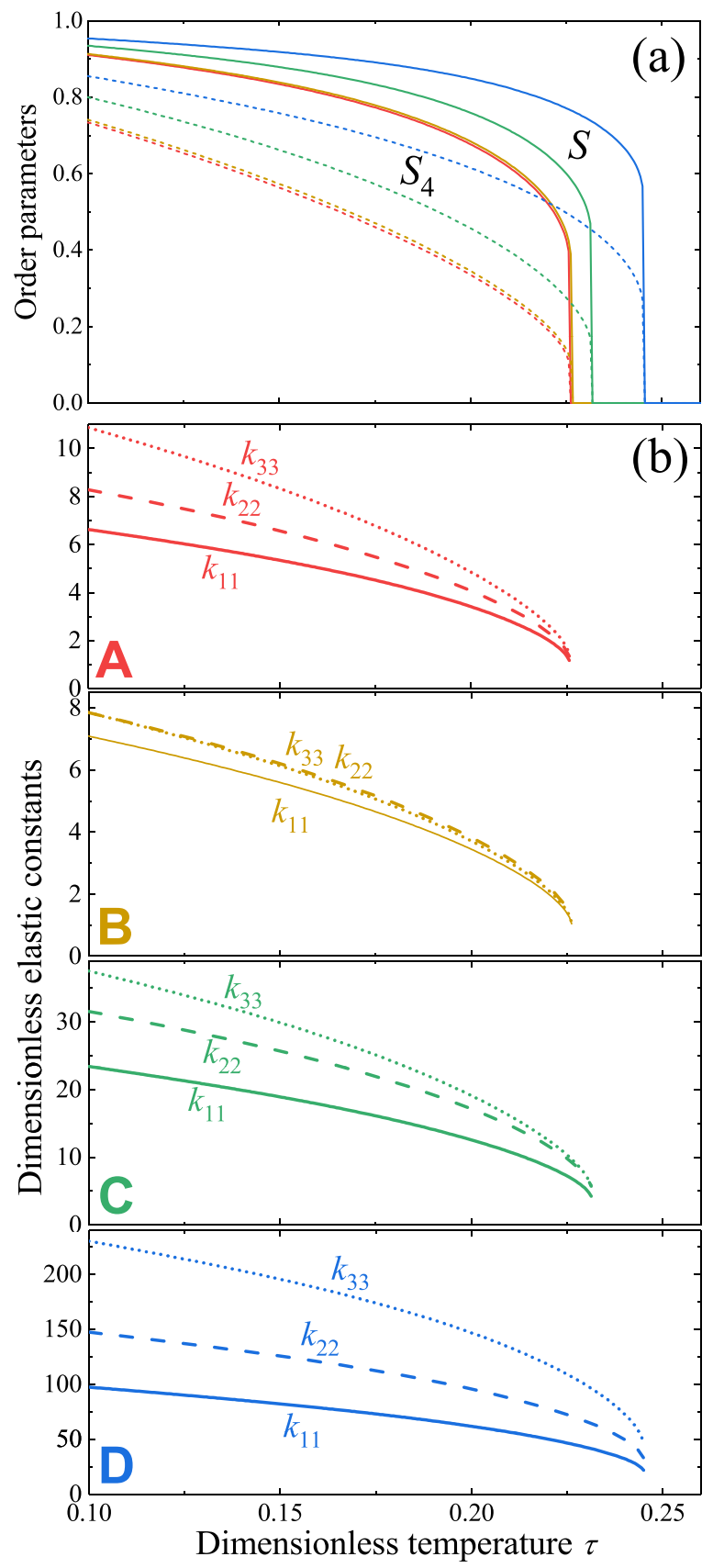

FIG. 5. Same as Fig. 4 for the modified G-B potential with $\mu=$ $1, v=2$. The four sets of parameters correspond to the points marked as A, B, C, and D in Fig. 2.

\section{ELASTICITY OF THE NEMATIC PHASE COMPOSED OF POLAR MOLECULES}

A. Contribution of polar intermolecular interactions to the free energy of the nematic phase

In the homogeneous uniaxial nematic state, the orientational one-particle distribution function $f_{1}(\mathbf{a}, \mathbf{b}, \mathbf{n})$ $=f\left((\mathbf{n} \cdot \mathbf{a})^{2},(\mathbf{n} \cdot \mathbf{b})^{2}\right)$ because the phase is nonpolar and hence there is only quadratic dependence on all orientational variables. Thus in the mean-field approximation, all polar molecular interactions that are described by the terms with 
odd $l$ and $k$ in the expansion Eq. (22) do not affect the homogeneous state.

In contrast, in the distorted nematic state, the polar part of intermolecular interactions gives rise to a small polar correction to the distribution function. In this paper, we restrict ourselves to uniaxial molecular interactions and hence the orientational distribution function depends only on the unit vector a along the primary molecular axis. In the first approximation, the orientational distribution function of the distorted nematic then can be expressed as:

$$
f_{1}(\mathbf{a}, \mathbf{n}(\mathbf{r}))=f(\mathbf{a} \cdot \mathbf{n}(\mathbf{r}))[1+h(\mathbf{a}, \mathbf{n}(\mathbf{r}))],
$$

where the distribution function $f(\mathbf{a} \cdot \mathbf{n})$ is even in $\mathbf{a}$ and $\mathbf{n}$, while the small polar correction $h(\mathbf{a}, \mathbf{n})$ is an odd function of $\mathbf{a}$.

This orientational distribution function together with the polar correction $h(\mathbf{a}, \mathbf{n}(\mathbf{r}))$ can be obtained by minimizing the free energy of the inhomogeneous nematic phase given by Eq. (21), where the distribution functions are more generally given by Eq. (52). Minimization of the free-energy results in the following expression:

$$
f\left[\mathbf{a}_{1} \cdot \mathbf{n}\left(\mathbf{r}_{1}\right)\right]\left[1+h\left(\mathbf{a}_{1}, \mathbf{n}\left(\mathbf{r}_{1}\right)\right)\right]=\frac{1}{Z} \exp \left\{-\frac{\rho}{k_{B} T} \int V\left(\mathbf{a}_{1}, \mathbf{r}_{12}, \mathbf{a}_{2}\right) f\left[\mathbf{a}_{2} \cdot \mathbf{n}\left(\mathbf{r}_{2}\right)\right]\left[1+h\left(\mathbf{a}_{2}, \mathbf{n}\left(\mathbf{r}_{2}\right)\right)\right] d \mathbf{a}_{2} d \mathbf{r}_{12}\right\},
$$

where

$$
Z=\int \exp \left\{-\frac{\rho}{k_{B} T} \int V\left(\mathbf{a}_{1}, \mathbf{r}_{12}, \mathbf{a}_{2}\right) f\left[\mathbf{a}_{2} \cdot \mathbf{n}\left(\mathbf{r}_{2}\right)\right]\left[1+h\left(\mathbf{a}_{2}, \mathbf{n}\left(\mathbf{r}_{2}\right)\right)\right] d \mathbf{a}_{2} d \mathbf{r}_{12}\right\} d \mathbf{a}_{1} .
$$

Substituting Eq. (53) back into Eq. (21) one expresses the free energy density as:

$$
\begin{aligned}
\mathcal{F}= & -\rho k_{B} T \ln Z-\frac{1}{2} \rho^{2} \int V\left(\mathbf{a}_{1}, \mathbf{r}_{12}, \mathbf{a}_{2}\right) f\left[\mathbf{a}_{2} \cdot \mathbf{n}\left(\mathbf{r}_{2}\right)\right]\left[1+h\left(\mathbf{a}_{2}, \mathbf{n}\left(\mathbf{r}_{2}\right)\right)\right] \\
& \times f\left[\mathbf{a}_{1} \cdot \mathbf{n}\left(\mathbf{r}_{1}\right)\right]\left[1+h\left(\mathbf{a}_{1}, \mathbf{n}\left(\mathbf{r}_{1}\right)\right)\right] d \mathbf{r}_{2} d \mathbf{a}_{1} d \mathbf{a}_{2} .
\end{aligned}
$$

Expanding the orientational distribution function $f\left(\mathbf{a}_{2} \cdot \mathbf{n}\left(\mathbf{r}_{2}\right)\right)$ in gradients of the director as:

$$
f\left[\mathbf{a}_{2} \cdot \mathbf{n}\left(\mathbf{r}_{2}\right)\right] \approx f\left[\mathbf{a}_{2} \cdot \mathbf{n}\left(\mathbf{r}_{1}\right)\right]+\left(\mathbf{r}_{12} \cdot \nabla\right) f\left[\mathbf{a}_{2} \cdot \mathbf{n}\left(\mathbf{r}_{1}\right)\right]+\frac{1}{2}\left(\mathbf{r}_{12} \cdot \nabla\right)^{2} f\left[\mathbf{a}_{2} \cdot \mathbf{n}\left(\mathbf{r}_{1}\right)\right]+\ldots,
$$

and accordingly expanding the free-energy density Eq. (55) in powers of the small function $h$, one can write the total free energy of the inhomogeneous nematic phase in the form:

$$
F=F_{h}+F_{d}+\int \mathcal{F}_{p}\left(\mathbf{r}_{1}\right) d \mathbf{r}_{1} .
$$

Here $F_{h}$ and $F_{d}$ are the free energies given by Eqs. (28) and (29) of nonpolar nematic considered above, while $\mathcal{F}_{p}$ describes the additional free energy of distortion:

$$
\begin{aligned}
\mathcal{F}_{p}\left(\mathbf{r}_{1}\right)= & -\frac{1}{2} \rho^{2} \int V\left(\mathbf{a}_{1}, \mathbf{r}_{12}, \mathbf{a}_{2}\right) f\left[\mathbf{a}_{2} \cdot \mathbf{n}\left(\mathbf{r}_{2}\right)\right] f\left[\mathbf{a}_{1} \cdot \mathbf{n}\left(\mathbf{r}_{1}\right)\right] h\left(\mathbf{a}_{2}, \mathbf{n}\left(\mathbf{r}_{2}\right)\right) h\left(\mathbf{a}_{1}, \mathbf{n}\left(\mathbf{r}_{1}\right)\right) d \mathbf{r}_{12} d \mathbf{a}_{2} d \mathbf{a}_{1} \\
& +\frac{1}{2} \rho^{2} \int V\left(\mathbf{a}_{1}, \mathbf{r}_{12}, \mathbf{a}_{2}\right) f\left[\mathbf{a}_{1} \cdot \mathbf{n}\left(\mathbf{r}_{1}\right)\right] h\left(\mathbf{a}_{2}, \mathbf{n}\left(\mathbf{r}_{2}\right)\right)\left(\mathbf{r}_{12} \cdot \nabla\right) f\left[\mathbf{a}_{2} \cdot \mathbf{n}\left(\mathbf{r}_{1}\right)\right] d \mathbf{r}_{12} d \mathbf{a}_{2} d \mathbf{a}_{1} \\
& -\frac{1}{2} \rho^{2} \int V\left(\mathbf{a}_{1}, \mathbf{r}_{12}, \mathbf{a}_{2}\right) f\left[\mathbf{a}_{1} \cdot \mathbf{n}\left(\mathbf{r}_{1}\right)\right] h\left(\mathbf{a}_{1}, \mathbf{n}\left(\mathbf{r}_{1}\right)\right)\left(\mathbf{r}_{12} \cdot \nabla\right) f\left[\mathbf{a}_{2} \cdot \mathbf{n}\left(\mathbf{r}_{1}\right)\right] d \mathbf{r}_{12} d \mathbf{a}_{2} d \mathbf{a}_{1} \\
& -\frac{\rho^{3}}{2 k_{B} T} \int f\left[\mathbf{a}_{1} \cdot \mathbf{n}\left(\mathbf{r}_{1}\right)\right]\left[\int V\left(\mathbf{a}_{1}, \mathbf{r}_{12}, \mathbf{a}_{2}\right) f\left[\mathbf{a}_{2} \cdot \mathbf{n}\left(\mathbf{r}_{1}\right)\right] h\left(\mathbf{a}_{2}, \mathbf{n}\left(\mathbf{r}_{2}\right)\right) d \mathbf{a}_{2} d \mathbf{r}_{12}\right]^{2} d \mathbf{a}_{1} .
\end{aligned}
$$

One notes that only the polar part of the intermolecular interaction potential contributes to all integral terms in Eq. (58).

Taking into account that the function $h(\mathbf{a}, \mathbf{n}(\mathbf{r}))$ is odd in $\mathbf{a}$, one concludes that the first term in Eq. (58) does not vanish only if the potential $V\left(\mathbf{a}_{1}, \mathbf{r}_{12}, \mathbf{a}_{2}\right)$ is odd both in $\mathbf{a}_{1}$ and $\mathbf{a}_{2}$ while the second term in Eq. (58) is nonzero only if the potential is odd both in $\mathbf{a}_{2}$ and $\mathbf{u}_{12}$. In a similar way, the third term does not vanish if the potential is odd both in $\mathbf{a}_{1}$ and $\mathbf{u}_{12}$. Finally, the last term in Eq. (58) does not vanish only if the potential is odd in $\mathbf{a}_{2}$ and even in $\mathbf{u}_{12}$.
Accordingly, it is convenient to present an arbitrary polar achiral potential of interaction between uniaxial molecules $V_{p}\left(\mathbf{a}_{1}, \mathbf{r}_{12}, \mathbf{a}_{2}\right)$ as a sum of the two parts:

$$
V_{p}\left(\mathbf{a}_{1}, \mathbf{r}_{12}, \mathbf{a}_{2}\right)=V_{p 1}\left(\mathbf{a}_{1}, \mathbf{r}_{12}, \mathbf{a}_{2}\right)+V_{p 2}\left(\mathbf{a}_{1}, \mathbf{r}_{12}, \mathbf{a}_{2}\right),
$$

where the potential $V_{p 1}\left(\mathbf{a}_{1}, \mathbf{r}_{12}, \mathbf{a}_{2}\right)$ is odd both in $\mathbf{a}_{1}$ and $\mathbf{a}_{2}$, while $V_{p 2}\left(\mathbf{a}_{1}, \mathbf{r}_{12}, \mathbf{a}_{2}\right)$ is odd in $\mathbf{r}_{12}$ and also in $\mathbf{a}_{1}$ or in $\mathbf{a}_{2}$. Both parts of the potential can be expanded in polar spherical invariants as in Eq. (22). For $V_{p 1}\left(\mathbf{a}_{1}, \mathbf{r}_{12}, \mathbf{a}_{2}\right)$, the indices $l$ and $k$ are odd and the index $m$ is even. In contrast, the expansion of the potential $V_{p 2}\left(\mathbf{a}_{1}, \mathbf{r}_{12}, \mathbf{a}_{2}\right)$ contains only invariants with odd $l$, odd $m$, and even $k$ or odd $k$, odd $m$, and even $l$. 


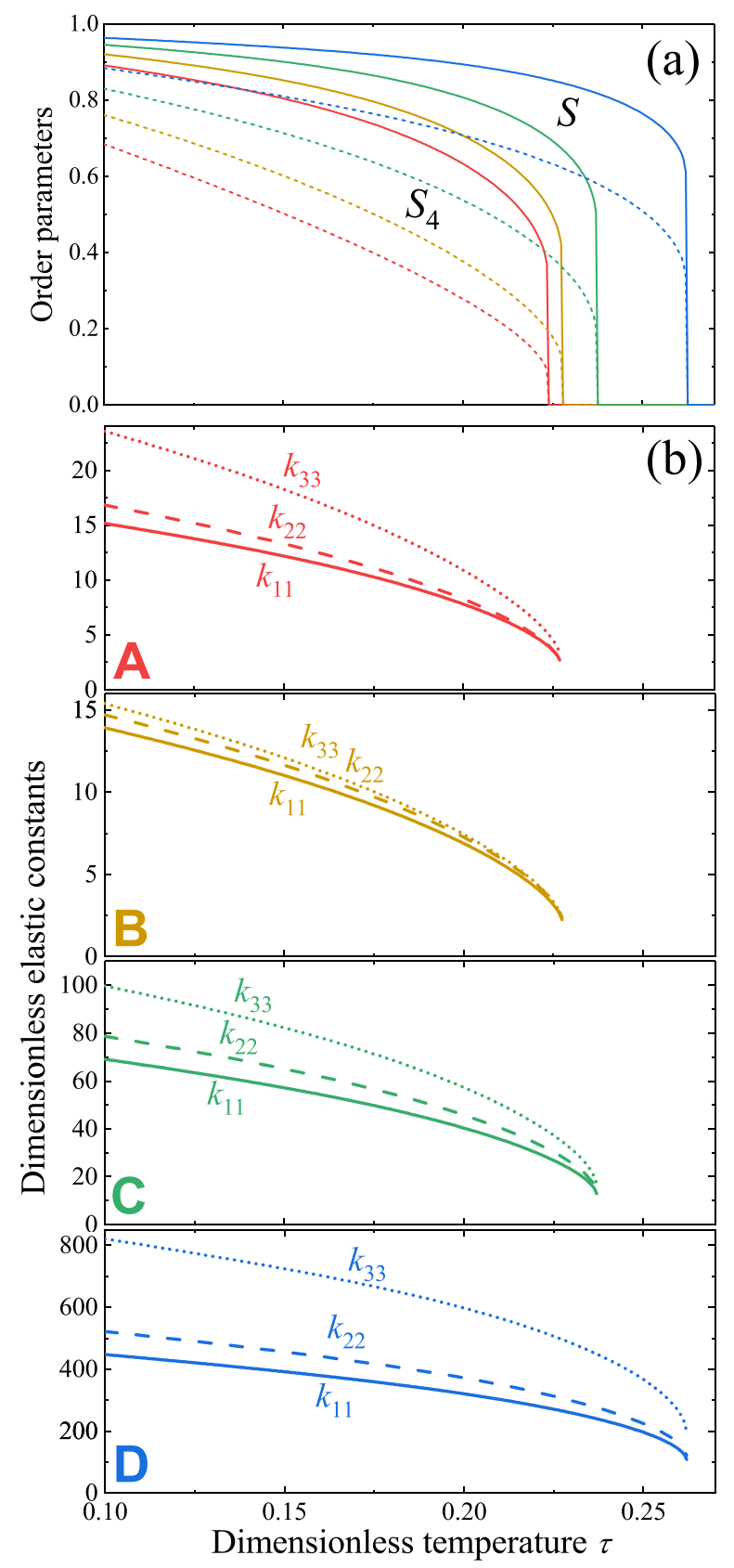

FIG. 6. Same as Fig. 4 for the modified G-B potential with $\mu=$ $1, v=3$. The four sets of parameters correspond to the points marked as A, B, C, and D in Fig. 3.

One concludes that the potential $V_{p 1}\left(\mathbf{a}_{1}, \mathbf{r}_{12}, \mathbf{a}_{2}\right)$ does not contribute to the second and third terms in Eq. (58) because it is even in the unit intermolecular vector $\mathbf{u}_{12}$ and hence these terms vanish after integration over $\mathbf{u}_{12}$. It contributes, however, to the first and last terms in Eq. (58). In a similar way, the potential $V_{p 2}\left(\mathbf{a}_{1}, \mathbf{r}_{12}, \mathbf{a}_{2}\right)$ which is odd in $\mathbf{u}_{12}$ does not contribute to the first and last terms in Eq. (58) providing a nonzero contribution to the second and third terms.

Taking into account, for simplicity, only the invariants of the first and second order in $\mathbf{a}_{1}$ and $\mathbf{a}_{2}$, one obtains the following approximate expressions:

$$
V_{p 1}\left(\mathbf{a}_{1}, \mathbf{r}_{12}, \mathbf{a}_{2}\right) \approx U_{101}\left(r_{12}\right) T^{101}\left(\mathbf{a}_{1}, \mathbf{u}_{12}, \mathbf{a}_{2}\right)
$$

$$
+U_{121}\left(r_{12}\right) T^{121}\left(\mathbf{a}_{1}, \mathbf{u}_{12}, \mathbf{a}_{2}\right)
$$

and

$$
\begin{aligned}
& V_{p 2}\left(\mathbf{a}_{1}, \mathbf{r}_{12}, \mathbf{a}_{2}\right) \\
& \approx U_{110}\left(r_{12}\right)\left[T^{110}\left(\mathbf{a}_{1}, \mathbf{u}_{12}, \mathbf{a}_{2}\right)-T^{011}\left(\mathbf{a}_{1}, \mathbf{u}_{12}, \mathbf{a}_{2}\right)\right] \\
& \quad+U_{112}\left(r_{12}\right)\left[T^{112}\left(\mathbf{a}_{1}, \mathbf{u}_{12}, \mathbf{a}_{2}\right)-T^{211}\left(\mathbf{a}_{1}, \mathbf{u}_{12}, \mathbf{a}_{2}\right)\right] .
\end{aligned}
$$

Substituting Eqs. (60) and (61) into Eq. (58) one notices that the unknown polar correction $h$ to the distribution function contributes to all integrals only via a vector:

$$
\mathbf{m}=\int \mathbf{a} f[\mathbf{a} \cdot \mathbf{n}(\mathbf{r})] h(\mathbf{r}, \mathbf{a}) d^{2} \mathbf{a},
$$

enabling one to express the free-energy density Eq. (58) as:

$$
\begin{aligned}
\mathcal{F}_{p}= & -2 \pi \rho^{2} m^{2} \mathcal{V}^{101} \\
& -2 \pi \rho^{2} \mathcal{W}^{112} S\{(\mathbf{m} \cdot \mathbf{n})(\nabla \cdot \mathbf{n})+[\mathbf{m} \cdot(\mathbf{n} \cdot \nabla) \mathbf{n}]\} \\
& -\frac{8 \pi^{2} \rho^{3}}{k_{B} T}\left(\mathcal{V}^{101}\right)^{2}\left\{S\left[(\mathbf{n} \cdot \mathbf{m})^{2}-\frac{m^{2}}{3}\right]+\frac{m^{2}}{3}\right\}
\end{aligned}
$$

where the constants $\mathcal{V}^{101}$ and $\mathcal{W}^{112}$ are defined by (A17) and (A19) respectively. It is worth mentioning that only the terms $U_{101}\left(r_{12}\right) T^{101}\left(\mathbf{a}_{1}, \mathbf{u}_{12}, \mathbf{a}_{2}\right)$ and $U_{112}\left(r_{12}\right) T^{112}\left(\mathbf{a}_{1}, \mathbf{u}_{12}, \mathbf{a}_{2}\right)$ provide nonzero contributions to Eq. (58).

The polar order parameter $\mathbf{m}$ can in principle be determined by minimization of the free-energy density Eq. (63). One notes, however, that the free-energy Eq. (63) possesses a minimum only within a certain range of parameters. Indeed, let us consider for simplicity the stability of the isotropic phase. In the isotropic phase $S=0$ and hence Eq. (63) can be written in the following simple form:

$$
\mathcal{F}_{p}=-2 \pi \rho^{2} \mathcal{V}^{101}\left(1+\frac{4 \pi \rho}{3 k_{B} T} \mathcal{V}^{101}\right) m^{2} .
$$

As the isotropic phase is nonpolar by definition, it should be stable with respect to the formation of spontaneous polar (e.g., ferroelectric) order specified by the order parameter $\mathbf{m}$, the energy density Eq. (64) must be non-negative. This requires the constant $\mathcal{V}^{101}$, which promotes the polar order, to be negative as well as the expression $\left(1+4 \pi \rho \mathcal{V}^{101} / 3 k_{B} T\right)$ to be positive, i.e., restricting $\left|\mathcal{V}^{101}\right|<3 k_{B} T / 4 \pi \rho$.

Minimizing the free-energy density Eq. (63) with respect to $\mathbf{m}$ yields its equilibrium value:

$$
\begin{aligned}
\mathbf{m}_{0}= & -\frac{\mathcal{W}^{112} S}{2 \mathcal{V}^{101}} \\
& \times\left\{\frac{\mathbf{n}(\nabla \cdot \mathbf{n})}{1+\frac{4 \pi \rho}{3 k_{B} T} \mathcal{V}^{101}(2 S+1)}+\frac{(\mathbf{n} \cdot \nabla) \mathbf{n}}{1+\frac{4 \pi \rho}{3 k_{B} T} \mathcal{V}^{101}(1-S)}\right\} .
\end{aligned}
$$

Finally, substituting $\mathbf{m}_{0}$ back into Eq. (63) one obtains the final expression for the polar correction to the free-energy density of deformation:

$$
\mathcal{F}_{p}=\frac{\pi \rho^{2}\left(\mathcal{W}^{112} S\right)^{2}}{2 \mathcal{V}^{101}}
$$




$$
\times\left\{\frac{(\nabla \cdot \mathbf{n})^{2}}{1+\frac{4 \pi \rho}{3 k_{B} T} \mathcal{V}^{101}(2 S+1)}+\frac{((\mathbf{n} \cdot \nabla) \mathbf{n})^{2}}{1+\frac{4 \pi \rho}{3 k_{B} T} \mathcal{V}^{101}(1-S)}\right\}
$$

Comparing with Eq. (13) and taking into account that ((n . $\nabla) \mathbf{n})^{2}=(\mathbf{n} \times \nabla \times \mathbf{n})^{2}$, one concludes that Eq. (66) describes the following corrections to splay and bend elastic constants which are determined by polar molecular shape:

$$
\begin{aligned}
\Delta K_{11} & =\frac{\pi \rho^{2}\left(\mathcal{W}^{112} S\right)^{2}}{2 \mathcal{V}^{101}} \gamma_{\|}, \\
\Delta K_{33} & =\frac{\pi \rho^{2}\left(\mathcal{W}^{112} S\right)^{2}}{2 \mathcal{V}^{101}} \gamma_{\perp},
\end{aligned}
$$

where

$$
\begin{aligned}
& \gamma_{\|}=\left[1+\frac{4 \pi \rho}{3 k_{B} T} \mathcal{V}^{101}(2 S+1)\right]^{-1}, \\
& \gamma_{\perp}=\left[1+\frac{4 \pi \rho}{3 k_{B} T} \mathcal{V}^{101}(1-S)\right]^{-1} .
\end{aligned}
$$

One notes that at small $S \ll 1$ both corrections are negative as $\mathcal{V}^{101}<0$ and $\left|\mathcal{V}^{101}\right|<3 k_{B} T / 4 \pi \rho$. Now let us consider the temperature variation of these corrections determined by the factors $\gamma_{\|}$and $\gamma_{\perp}$. At $S=0$, they are equal and positive $\gamma_{\|}=$ $\gamma_{\perp}=\left(1+4 \pi \rho \mathcal{V}^{101} / 3 k_{B} T\right)^{-1}$. However, the variation of $\gamma_{\|}$ with the decreasing temperature is completely different from that of $\gamma_{\perp}$. Indeed, $\gamma_{\perp}$ remains positive for all temperatures and values of $S$ and approaches unity in the limit of perfect orientational order $S=1$. Thus the polar correction to $K_{33}$ is generally small as long as the coupling constant $\mathcal{W}^{112}$ remains relatively small. In contrast, $\gamma_{\|}$increases with the decreasing temperature and diverges at some temperature $T=T_{\mathrm{spl}}$ when $4 \pi \rho\left|\mathcal{V}^{101}\right|\left[2 S\left(T_{\mathrm{spl}}\right)+1\right]=3 k_{B} T_{\mathrm{spl}}$.

Thus the polar correction to the splay elastic constant is negative and its absolute value increases with the decreasing temperature diverging at $T=T_{\text {spl }}$. Formally at $T=T_{\text {spl }}$ the nematic phase (if it still exists in this temperature range) looses its stability with respect to the homogeneous polar order. However, this phase transition is preempted by an elastic instability because the absolute value of the negative polar correction $\Delta K_{11}$ grows very fast in the pretransitional region above $T_{\text {spl }}$ and at some temperature $T_{0}>T_{\text {spl }}$ the total splay elastic constant (i.e., the sum of polar and nonpolar contributions) vanishes and the homogeneous nematic phase looses its stability with respect to the inhomogeneous director distribution. This may result in the formation of the splay-bend phase.

\section{B. Elastic constants of polar Gay-Berne nematic}

For a quantitative illustration of the effect of polar molecular shape on the elasticity of the nematic phase we consider a polar modification of the G-B potential (44) by introducing an additional factor to the anisotropic interaction range (45) which has the qualitative meaning of the closest distance of approach between the two molecules:

$$
\begin{aligned}
& \sigma\left(\mathbf{a}_{1}, \mathbf{u}, \mathbf{a}_{2}\right) \\
& \quad=\left[1-\eta\left(4-p^{2}\right)(2+p) p^{2}\right]
\end{aligned}
$$

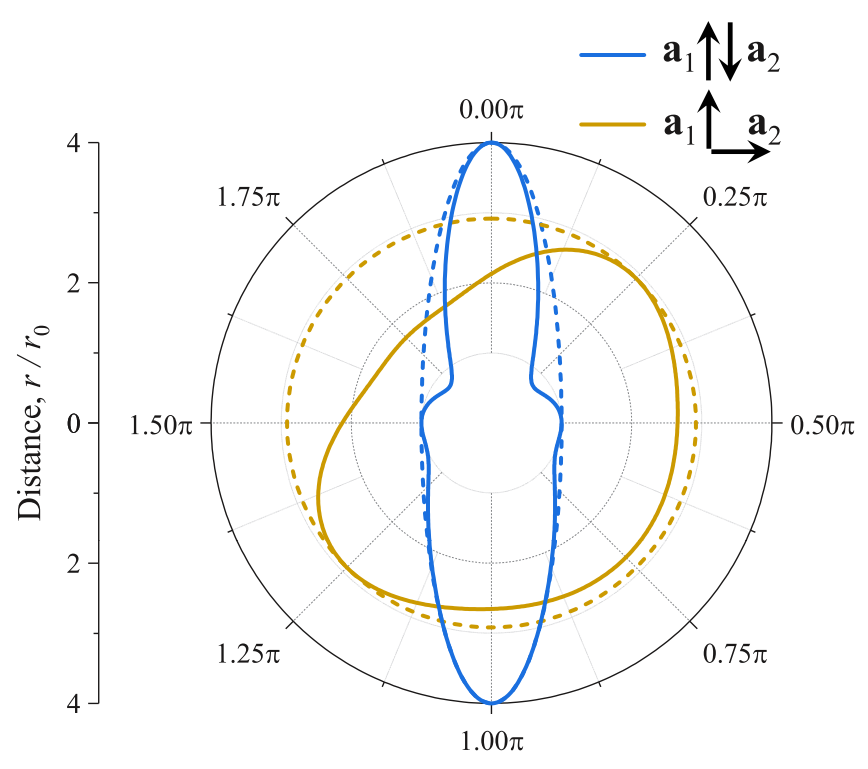

FIG. 7. Cuts of the range (71) of polar G-B potential with $\eta=$ 0.03 (solid) and $\eta=0$ (dashed) by the plane containing the main molecular axes $\mathbf{a}_{1,2}$ for their antiparallel and perpendicular orientations. The relative molecular elongation is $\kappa=4$.

$$
\times\left\{1-\frac{\chi}{2}\left[\frac{\left(\mathbf{u} \cdot \mathbf{a}_{1}+\mathbf{u} \cdot \mathbf{a}_{2}\right)^{2}}{1+\chi \mathbf{a}_{1} \cdot \mathbf{a}_{2}}+\frac{\left(\mathbf{u} \cdot \mathbf{a}_{1}-\mathbf{u} \cdot \mathbf{a}_{2}\right)^{2}}{1-\chi \mathbf{a}_{1} \cdot \mathbf{a}_{2}}\right]\right\}^{-1 / 2}
$$

The added factor depends on the molecular axes $\mathbf{a}_{1}, \mathbf{a}_{2}$ and the intermolecular unit vector $\mathbf{u}$ via the combination $p=$ $\left(\mathbf{u} \cdot \mathbf{a}_{1}-\mathbf{u} \cdot \mathbf{a}_{2}\right)$. The dependence on $p$ allows the potential to retain its symmetry with respect to the molecular permutation $\left(\mathbf{a}_{1} \leftrightarrow \mathbf{a}_{2}\right.$ and $\left.\mathbf{u} \leftrightarrow-\mathbf{u}\right)$. At the same time, it introduces the so-called head-tail asymmetry with respect to the flipping of the main axis of each molecule, $\mathbf{a}_{1} \leftrightarrow-\mathbf{a}_{1}$ or $\mathbf{a}_{2} \leftrightarrow-\mathbf{a}_{2}$.

The introduced polarity is quantitatively characterized by the parameter $\eta$ and the particular polynomial dependence on $p$ ensures that the range is not an even function of $p$ but remains fixed for certain molecular orientations corresponding to $p=0, \pm 2$. For instance, the polarity does not affect the interaction range of the molecules with parallel main axes, $\mathbf{a}_{1}=\mathbf{a}_{2}$. However, as shown in Fig. 7, sufficiently small values of $\eta$ result in a noticeable asymmetry of the interaction range for molecules aligned in an antiparallel way or perpendicular to each other.

Using the polar G-B potential one can evaluate the elastic constants (49) and (51) together with their polar corrections given by the following dimensionless expressions:

$$
\begin{aligned}
k_{11}^{(p)} & =\frac{\pi\left(w^{112} S\right)^{2}}{2 v^{101}\left[1+4 \pi v^{101}(2 S+1)\left(3 \tau\left|v^{202}\right|\right)^{-1}\right]}, \\
k_{33}^{(p)} & =\frac{\pi\left(w^{112} S\right)^{2}}{2 v^{101}\left[1+4 \pi v^{101}(1-S)\left(3 \tau\left|v^{202}\right|\right)^{-1}\right]},
\end{aligned}
$$

where the dimensionless parameter $w^{112}=\mathcal{W}^{112}\left(\varepsilon_{0} r_{0}^{4}\right)^{-1}$ is defined similarly to $u^{l m k}$ and $v^{l m k}$ introduced in Sec. V. The analytical integration over the intermolecular distance yields 

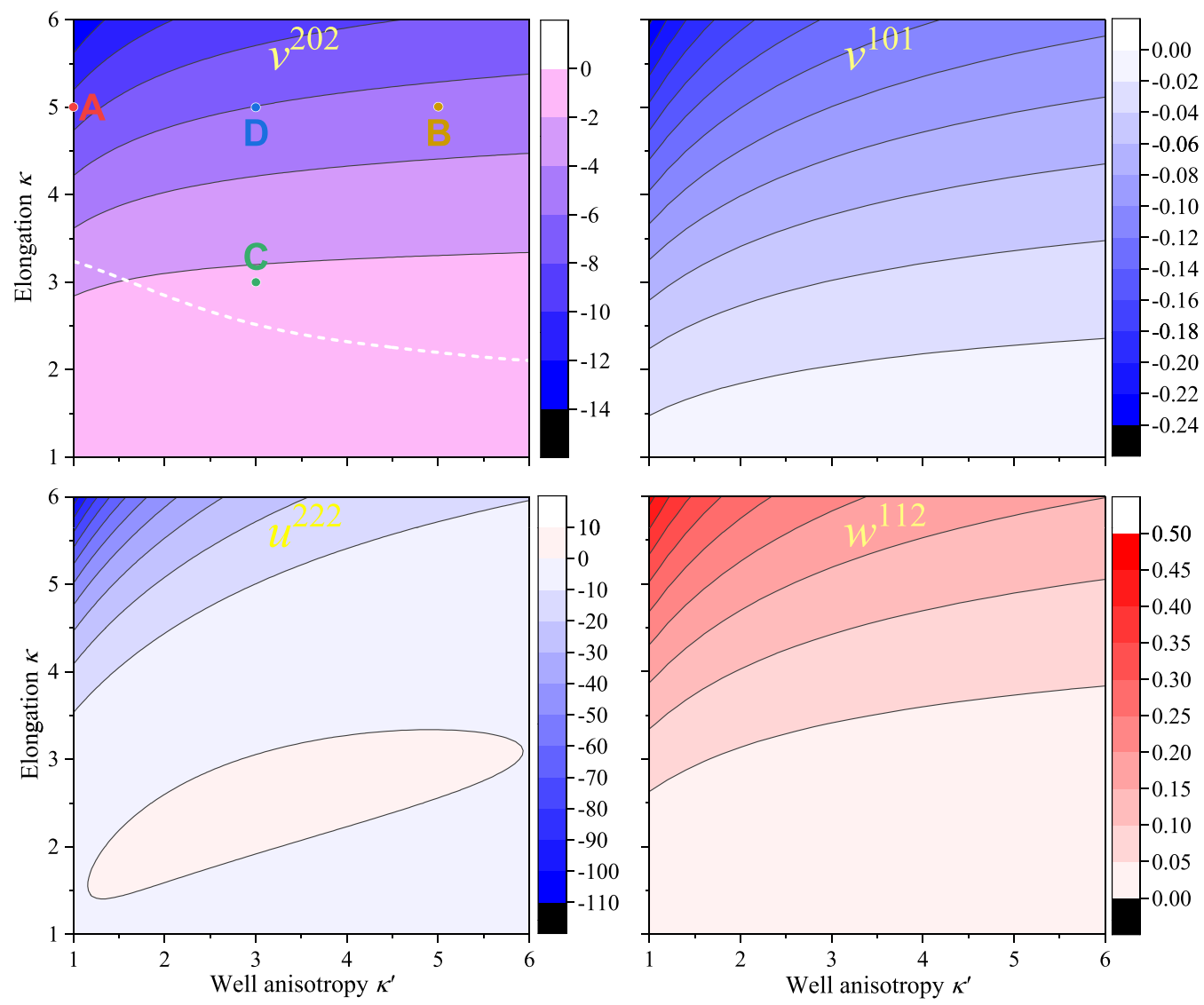

FIG. 8. Colormaps of dimensionless parameters of the expansion in spherical invariants of the modified polar G-B pair potential with $\mu=1, v=3$, and $\eta=0.005$. In the $v^{202}$ plot the four exemplary points $\mathrm{A}, \mathrm{B}, \mathrm{C}$, and D are shown for which we evaluate the elastics constants in Fig. 9 and the white dashed line shows the border of the area with $K_{11}$ singularity. The colormaps of not shown parameters are marginally affected by $\eta$ and remain practically identical to those in Fig. 3.

the orientational integral:

$$
\begin{aligned}
\mathcal{W}^{112}= & \frac{-16 \pi^{2}}{495} r_{0}^{4} \int d \mathbf{a}_{1} d \mathbf{a}_{2} T^{112}\left(\mathbf{a}_{1}, \mathbf{u}, \mathbf{a}_{2}\right) \\
& \times \varepsilon\left(\mathbf{a}_{1}, \mathbf{u}, \mathbf{a}_{2}\right)\left[65+124 \sigma\left(\mathbf{a}_{1}, \mathbf{u}, \mathbf{a}_{2}\right)\right. \\
& \left.+162 \sigma^{2}\left(\mathbf{a}_{1}, \mathbf{u}, \mathbf{a}_{2}\right)+144 \sigma^{3}\left(\mathbf{a}_{1}, \mathbf{u}, \mathbf{a}_{2}\right)\right],
\end{aligned}
$$

which has to be evaluated numerically.

We have employed the modified G-B potential with $\mu=1$ and $v=3$, add a weak polarity of the molecular shape specified by the parameter $\eta=0.005$ and calculated numerically all dimensionless parameters of the expansion of such a potential which enter the expressions for the elastic constants. It appears that the effect of the weak molecular polarity $\eta$ on the most of the parameters $u^{l m k}$ and $v^{l m k}$ which determine the nonpolar contributions to the elastic constants (49)-(51) is practically negligible apart from the parameter $u^{222}$. The latter, as discussed above and in Appendix D, arises as a result of partial cancellation of several large contributions, and the polar modification of the potential noticeably affects this balance. In Fig. 8 we present the colormap of the parameter $v^{202}$ which is practically unaffected by weak polarity $\eta$, the colormap of the parameter $u^{222}$ which is slightly altered by the polarity (compare with the similar plot in Fig. 3), as well as of the two additional expansion coefficients $v^{101}$ and $w^{112}$ which are determined by the molecular polarity.

In Fig. 9, we present the temperature variation of the elastic constants of polar nematics with the polar contributions (72) and (73) taken into account. One can readily see that in the case of weak polarity, the temperature dependencies of the twist and bend elastic constants remain practically unaffected. At the same time, the splay elastic constant $k_{11}$ behaves in a completely different way: First, it increases with the decreasing temperature similarly to $k_{33}$ and $k_{22}$ but then reaches a maximum and rapidly decreases until vanishing at some temperature $T_{0}$ which is different for different parameters of the interaction potential. One notes that the homogeneous nematic phase may be stable only in the temperature range between the nematic-isotropic transition temperature $T_{\mathrm{NI}}$ and $T_{0}$ provided that $T_{\mathrm{NI}}>T_{0}$. For some values of the parameters, the instability temperature $T_{0}$ may be formally higher than $T_{\mathrm{NI}}$ and then there is no stable nematic phase at all. This is illustrated in Fig. 8 where all points on the white dashed line correspond to $T_{\mathrm{NI}}=T_{0}$. Below this line, $T_{\mathrm{NI}}<T_{0}$, and hence all combinations of parameters do not correspond to a stable homogeneous nematic phase. Note that this area covers a substantial part of the $\kappa-\kappa^{\prime}$ plane. It is truly remarkable how a very weak polar interaction which corresponds to less than $1 \%$ of the total intermolecular interaction po- 


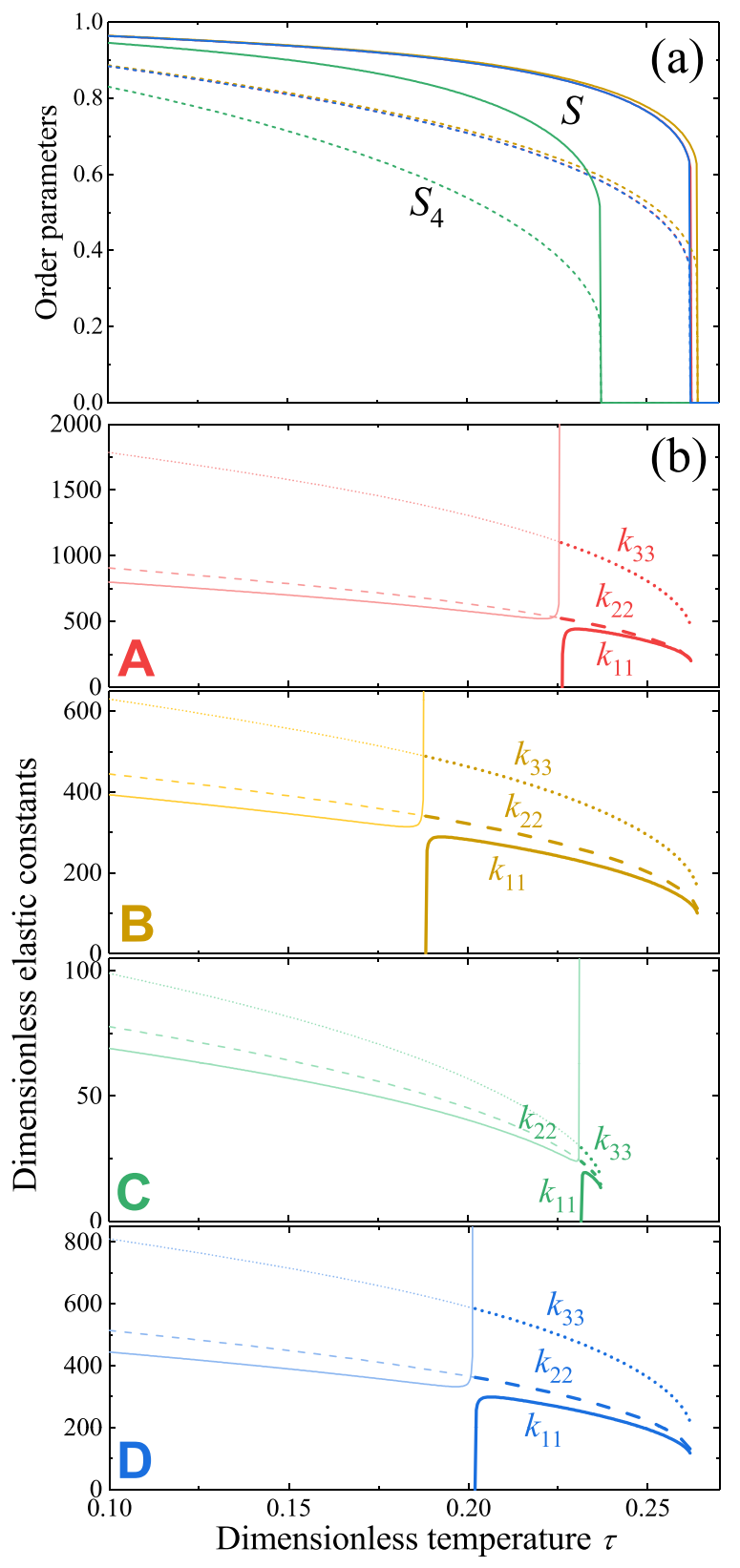

FIG. 9. Same as Fig. 6 for the modified polar G-B potential with $\mu=1, v=3$, and $\eta=0.005$. The four sets of parameters correspond to the points marked as A, B, C, and D in Fig. 8.

tential may completely destabilize the nematic phase at all temperatures.

\section{DISCUSSION}

In this paper we have used a novel approach to the evaluation of the elastic constants of nematic LCs. The distortion free energy of the nematic phase has been expressed in terms of the orientational deformations tensor $\gamma_{i j}=$ $\epsilon_{i l k} n_{l} \nabla_{j} n_{k}$ which specifies the pure rotation of the director and explicitly accounts for the nonpolar symmetry of the nematic phase. The general fourth rank elasticity tensor of the nematic phase has been expressed as a sum of five independent terms, and it has been shown that only three terms contribute to the elastic constants. As a result, the Frank elastic constants have been rigorously expressed in terms of the three corresponding coefficients. One notes that these coefficients which have not been considered in the literature before, have a physical meaning. For example, the constants $\mathcal{K}_{12}$ and $\mathcal{K}_{3}$ determine the difference between $K_{11}$ and $K_{22}$, and $K_{11}$ and $K_{33}$, respectively. Finally, explicit expressions for the elastic constants have been obtained as the contractions of the elasticity tensor with the appropriate tensors which depend on the components of the director.

Approximate expressions for the elastic constants have been derived using the molecular-field approximation in the molecular theory of nematic LCs. The intermolecular interaction potential is expanded in the complete set of functions-the so-called spherical invariants. As a result, the elastic constants of the nematic phase are expressed as sums of the contributions which depend on the corresponding expansion coefficients and on the orientational order parameters of the increasing order. In practice, such a sum can be truncated after the first few terms because the remaining terms depend on the higher order parameters which decrease rapidly with the increasing rank. It has also been shown that the elasticity tensor of the nematic phase, obtained in this paper can be transformed into the form which exactly corresponds to the general result derived in Ref. [22] provided the molecular-field approximation is employed. One notes, however, that the expressions for the elastic constants, obtained, for example, in Ref. [22] depend on the derivatives of the orientational distribution function which are to be evaluated separately, and thus the elastic constants are not expressed as explicit ensemble averages. In contrast, in the present theory the intermolecular potential is expanded in spherical invariants and each term is explicitly averaged with the distribution functions. Therefore, the theory establishes a direct approximate relationship between Frank elastic constants and the intermolecular interaction potential and the order parameters.

One notes that this approach can in principle be used for any interaction potential which is available in the analytical or in the numerical form. For illustration, we have calculated numerically all elastic constants for the model nematic LC composed of molecules interacting via the popular G-B potential. Both the classical version of the G-B potential and two its modifications have been used and the elastic constants have been calculated as functions of temperature, for different molecular elongation and anisotropy of the potential well. Surprisingly, the model nematic phase with the classical G-B interaction appears to be unstable with respect to the director deformations in the broad range of the model parameters. Moreover, even in the stable nematic phase the largest elastic constant is $K_{22}$ which is in contradiction with typical experimental data for nematic LCs composed of prolate molecules. In contrast, for the two modified versions of the G-B potential [32-34], the nematic phase is always stable and $K_{33}$ is the largest elastic constant as one expects. It has also been shown that the difference between the elastic constants is increasing with the increasing molecular elongation. Thus the results indicate that Frank elastic constants are significantly more sensitive to the details of the intermolecular 
interactions than the order parameters, and some particular interactions may lead to the elastic instabilities in the nematic phase.

The molecular theory of elasticity of the nematic phase has also been generalized to account for the polar molecular shape. It has been taken into consideration that the orientational distribution function of polar molecules in the nematic phase with inhomogeneous director distribution contains a small correction which is proportional to bend and splay deformation vectors $[8,14]$. This means that even in the system of uniaxial polar molecules the distribution function does not have the simple form $f(\mathbf{a}, \mathbf{r})=f[\mathbf{a} \cdot \mathbf{n}(\mathbf{r})]$ as assumed in the molecular theory of nematic LCs composed of nonpolar molecules. The polar correction has been determined by minimization of the free energy of the inhomogeneous nematic phase taking into account the gradient terms and polar intermolecular interactions. A small polar correction to the orientational distribution function gives rise to the corresponding corrections to splay and bend elastic constants which appear to be negative throughout the range of the stable nematic phase. It has been shown that the behavior of these corrections is completely different. In the case of uniaxial polar molecules, considered in this paper, the polar correction to the bend elastic constant is generally small for weakly polar molecules and its absolute value decreases with the decreasing temperature. In contrast, the absolute value of the correction to the splay elastic constant rapidly increases with the decreasing temperature and may even diverge at some temperature $T_{\mathrm{spl}}$. At this temperature, the nematic phase looses its stability with respect to the formation of the homogeneous polar (e.g., ferroelectric) order.

It is interesting to note, however, that this polar instability can never occur in the conventional nematic phase because it is preempted by another structural instability. In the pretransitional region above $T_{\mathrm{spl}}$ the absolute value of the negative polar correction $\Delta K_{11}$ can be very large and at some temperature $T_{0}>T_{\text {spl }}$ the total splay elastic constant $K_{11}$ (the sum of the positive nonpolar and negative polar contributions) vanishes. This results in an instability of the homogeneous nematic phase with respect to an inhomogeneous distribution of the director and may be considered as a mechanism of the transition into the splay-bend phase $[1,2,3]$.

One concludes that the homogeneous nematic phase may be stable only in the temperature range between the nematic-isotropic transition temperature $T_{\mathrm{NI}}$ and the instability temperature $T_{0}$, and both these temperatures strongly depend on the parameters of the interaction potential. It has been shown that in some areas of the parameters space $T_{0}$ is higher than $T_{\mathrm{NI}}$ and hence the systems with the corresponding values of the parameters do not exhibit any stable homogeneous nematic phase. Thus even a weak polar intermolecular interaction may completely destabilize the nematic phase at all temperatures.

The theory developed in this paper may also be generalized to describe elasticity of nematic LCs composed of biaxial and polar bent-core molecules which may exhibit the twist-bend phase. Bent-core molecules can be modeled, for example, by two G-B interaction cites. The corresponding theory will be presented in our future publication.

\section{ACKNOWLEDGMENTS}

The work of M.A.O. was carried out within the State Program of TIPS RAS. The work of M.V.G. and A.A.A. is supported by the Ministry of Science and Higher Education of the Russian Federation within the State assignment of FSRC "Crystallography and Photonics" RAS.

\section{APPENDIX A: INVARIANTS $T^{l m k}$}

The first several functions $T^{l m k}$ read as:

$$
\begin{gathered}
T^{101}\left(\mathbf{a}_{1}, \mathbf{u}_{12}, \mathbf{a}_{2}\right)=\left(\mathbf{a}_{1} \cdot \mathbf{a}_{2}\right) \\
T^{110}\left(\mathbf{a}_{1}, \mathbf{u}_{12}, \mathbf{a}_{2}\right)=\left(\mathbf{a}_{1} \cdot \mathbf{u}_{12}\right) \\
T^{011}\left(\mathbf{a}_{1}, \mathbf{u}_{12}, \mathbf{a}_{2}\right)=\left(\mathbf{a}_{2} \cdot \mathbf{u}_{12}\right) \\
T^{121}\left(\mathbf{a}_{1}, \mathbf{u}_{12}, \mathbf{a}_{2}\right)=3\left(\mathbf{a}_{1} \cdot \mathbf{u}_{12}\right)\left(\mathbf{a}_{2} \cdot \mathbf{u}_{12}\right)-\left(\mathbf{a}_{1} \cdot \mathbf{a}_{2}\right), \\
T^{112}\left(\mathbf{a}_{1}, \mathbf{u}_{12}, \mathbf{a}_{2}\right)=3\left(\mathbf{a}_{1} \cdot \mathbf{a}_{2}\right)\left(\mathbf{a}_{2} \cdot \mathbf{u}_{12}\right)-\left(\mathbf{a}_{1} \cdot \mathbf{u}_{12}\right), \\
T^{211}\left(\mathbf{a}_{1}, \mathbf{u}_{12}, \mathbf{a}_{2}\right)=3\left(\mathbf{a}_{1} \cdot \mathbf{a}_{2}\right)\left(\mathbf{a}_{1} \cdot \mathbf{u}_{12}\right)-\left(\mathbf{a}_{2} \cdot \mathbf{u}_{12}\right), \\
T^{220}\left(\mathbf{a}_{1}, \mathbf{u}_{12}, \mathbf{a}_{2}\right)=P_{2}\left(\mathbf{a}_{1} \cdot \mathbf{u}_{12}\right) \\
=\frac{3}{2}\left(\mathbf{a}_{1} \cdot \mathbf{u}_{12}\right)^{2}-\frac{1}{2}, \\
T^{202}\left(\mathbf{a}_{1}, \mathbf{u}_{12}, \mathbf{a}_{2}\right)=P_{2}\left(\mathbf{a}_{1} \cdot \mathbf{a}_{2}\right) \\
T^{404}\left(\mathbf{a}_{2}\right)^{2}-\frac{1}{2}, \\
T^{422}\left(\mathbf{a}_{1}, \mathbf{u}_{12}, \mathbf{a}_{2}\right) \quad\left(\mathbf{u}_{12}, \mathbf{a}_{2}\right)=P_{4}\left(\mathbf{a}_{1} \cdot \mathbf{a}_{2}\right) \\
\left.=\frac{35}{8}\left(\mathbf{a}_{1} \cdot \mathbf{a}_{2}\right)^{2}\left(\mathbf{a}_{12} \cdot \mathbf{u}_{12}\right)^{2}, \mathbf{a}_{2}\right)=P_{2}\left(\mathbf{a}_{2} \cdot \mathbf{u}_{12}\right) \\
-\frac{5}{2}\left(\mathbf{a}_{1} \cdot \mathbf{a}_{2}\right)\left(\mathbf{a}_{2} \cdot \mathbf{u}_{12}\right)\left(\mathbf{a}_{1} \cdot \mathbf{u}_{12}\right)+\frac{1}{4}\left(\mathbf{u}_{12} \cdot \mathbf{a}_{2}\right)^{2} \\
-\frac{5}{8}\left(\mathbf{u}_{12} \cdot \mathbf{a}_{1}\right)^{2}-\frac{5}{8}\left(\mathbf{a}_{1} \cdot \mathbf{a}_{2}\right)^{2}+\frac{1}{8}, \\
\left.T^{222}\left(\mathbf{a}_{1}, \mathbf{u}_{12}, \mathbf{a}_{2}\right) \quad \frac{9}{2}, \mathbf{a}_{2}\right)^{4}-\frac{30}{8}\left(\mathbf{a}_{1} \cdot \mathbf{a}_{2}\right)^{2}+\frac{3}{8}, \\
=\frac{9}{2}\left(\mathbf{a}_{1} \cdot \mathbf{a}_{2}\right)\left(\mathbf{a}_{2} \cdot \mathbf{u}_{12}\right)\left(\mathbf{a}_{1} \cdot \mathbf{u}_{12}\right) \\
-\frac{3}{2}\left(\mathbf{a}_{1} \cdot \mathbf{a}_{2}\right)^{2}-\frac{3}{2}\left(\mathbf{a}_{1} \cdot \mathbf{u}_{12}\right)^{2}-\frac{3}{2}\left(\mathbf{u}_{12} \cdot \mathbf{a}_{2}\right)^{2}+1,
\end{gathered}
$$

and

$$
T^{224}\left(\mathbf{a}_{1}, \mathbf{u}_{12}, \mathbf{a}_{2}\right)=T^{422}\left(\mathbf{a}_{2}, \mathbf{u}_{12}, \mathbf{a}_{1}\right) .
$$

The functions are mutually orthogonal in a sense that

$$
\begin{aligned}
& \int T^{l m k}\left(\mathbf{a}_{1}, \mathbf{u}_{12}, \mathbf{a}_{2}\right) T^{l^{\prime} m^{\prime} k^{\prime}}\left(\mathbf{a}_{1}, \mathbf{u}_{12}, \mathbf{a}_{2}\right) d \mathbf{a}_{1} d \mathbf{a}_{2} \\
& =\delta_{l l^{\prime}} \delta_{m m^{\prime}} \delta_{k k^{\prime}}\left\|T^{l m k}\right\|,
\end{aligned}
$$

but not properly normalized, as $\left\|T^{101}\right\|=\left\|T^{110}\right\|=$ $\left\|T^{011}\right\|=\frac{16}{3} \pi^{2}, \quad\left\|T^{121}\right\|=\left\|T^{112}\right\|=\left\|T^{211}\right\|=\frac{32}{3} \pi^{2}$, 
$\left\|T^{220}\right\|=\left\|T^{202}\right\|=\left\|T^{022}\right\|=\frac{16}{5} \pi^{2},\left\|T^{222}\right\|=\frac{56}{25} \pi^{2}$, and $\left\|T^{224}\right\|=\left\|T^{242}\right\|=\left\|T^{422}\right\|=\frac{56}{45} \pi^{2},\left\|T^{404}\right\|=\frac{16}{9} \pi^{2}$.

Therefore, expansion of an arbitrary pair interaction potential $U\left(\mathbf{a}_{1}, \mathbf{r}, \mathbf{a}_{2}\right)$ reads as:

$$
U\left(\mathbf{a}_{1}, \mathbf{r}, \mathbf{a}_{2}\right)=\sum_{l m k} U_{l m k}(r) T^{l m k}\left(\mathbf{a}_{1}, \frac{\mathbf{r}}{r}, \mathbf{a}_{2}\right),
$$

with the coefficients obtained as

$$
\begin{aligned}
U_{l m k}(r)= & \frac{1}{\left\|T^{l m k}\right\|} \\
& \times \int U\left(\mathbf{a}_{1}, \mathbf{r}, \mathbf{a}_{2}\right) T^{l m k}\left(\mathbf{a}_{1}, \frac{\mathbf{r}}{r}, \mathbf{a}_{2}\right) d \mathbf{a}_{1} d \mathbf{a}_{2} .
\end{aligned}
$$

Note that upon integration, the right-hand side is independent of the direction of $\mathbf{r}$.

If a potential is even with respect to the permutation $1 \leftrightarrow 2$ of interacting particles, then certain coefficients are interrelated. In particular, $U_{110}(r)=-U_{011}(r), U_{112}(r)=-U_{211}(r)$, $U_{220}(r)=U_{022}(r)$, and $U_{422}(r)=U_{224}(r)$.

The contributions to the homogeneous nematic free energy is expressed by the integrals

$$
\mathcal{V}^{l m k}=\int_{0}^{\infty} d r r^{2} U_{l m k}(r),
$$

the elastic constants of nonpolar nematic are determined by the integrals

$$
\mathcal{U}^{l m k}=\int_{0}^{\infty} d r r^{4} U_{l m k}(r),
$$

while the elasticity of nematics composed of polar molecules involves also the integrals

$$
\mathcal{W}^{l m k}=\int_{0}^{\infty} d r r^{3} U_{l m k}(r) .
$$

\section{APPENDIX B: COMPARISON WITH THE GENERAL EXPRESSIONS OF PONIEWIERSKI AND STECKI}

Poniewierski and Stecki [22] have considered the orientational elasticity of the nematic phase composed of uniaxial nonpolar molecules by introducing another elasticity tensor $M_{j q t s}$ that presents the corresponding part of the distortion free-energy density as

$$
\mathcal{F}_{d}=\frac{1}{2} k_{B} T M_{j q t s} \frac{\partial n_{t}}{\partial x_{j}} \frac{\partial n_{s}}{\partial x_{q}} .
$$

They have obtained the following exact expression for this elasticity tensor:

$$
\begin{aligned}
M_{j q t s}= & \frac{1}{2} \rho^{2} \int r_{12, j} r_{12, q} C_{2}\left(\mathbf{a}_{1}, \mathbf{r}_{12}, \mathbf{a}_{2}\right) a_{2, t} a_{1, s} \\
& \times \frac{\partial f\left(\mathbf{a}_{1} \cdot \mathbf{n}\right)}{\partial\left(\mathbf{a}_{1} \cdot \mathbf{n}\right)} \frac{\partial f\left(\mathbf{a}_{2} \cdot \mathbf{n}\right)}{\partial\left(\mathbf{a}_{2} \cdot \mathbf{n}\right)} d \mathbf{r}_{12} d \mathbf{a}_{1} d \mathbf{a}_{2},
\end{aligned}
$$

where $C_{2}\left(\mathbf{a}_{1}, \mathbf{r}_{12}, \mathbf{a}_{2}\right)$ is the direct pair correlation function of the homogeneous nematic phase. The Frank elastic constants are then expressed in terms of the components of the tensor $M_{j q t s}$ as $K_{11}=M_{x x x x}, K_{22}=M_{x y x y}$, and $K_{33}=M_{x z x z}$. It can readily be shown that exactly the same expression can also be derived from the above Eq. (31) in terms of the molecular-field approximation.

Indeed, by comparing Eq. (B1) with Eq. (31) one identifies this elasticity tensor in the form:

$$
\begin{aligned}
M_{j q t s}^{\mathrm{MF}} & =-\frac{\rho^{2}}{2 k_{B} T} \sum_{l m k} S_{l} S_{k} \int d \mathbf{r}_{12} r_{12, j} r_{12, q} U_{l m k}\left(r_{12}\right)\left[\frac{\partial^{2} T^{l m k}}{\partial n_{2, t} \partial n_{1, s}}\left(\mathbf{n}_{1}, \mathbf{u}_{12}, \mathbf{n}_{2}\right)\right]_{\mathbf{n}_{2}=\mathbf{n}_{1}=\mathbf{n}} \\
& =-\frac{\rho^{2}}{2 k_{B} T} \int d \mathbf{r}_{12} r_{12, j} r_{12, q}\left[\frac{\partial^{2}}{\partial n_{2, t} \partial n_{1, s}}\left(\sum_{l m k} S_{l} S_{k} U_{l m k}\left(r_{12}\right) T^{l m k}\left(\mathbf{n}_{1}, \mathbf{u}_{12}, \mathbf{n}_{2}\right)\right)\right]_{\mathbf{n}_{2}=\mathbf{n}_{1}=\mathbf{n}} \\
& =-\frac{\rho^{2}}{2 k_{B} T}\left[\frac{\partial^{2}}{\partial n_{2, t} \partial n_{1, s}}\left(\int d \mathbf{r}_{12} r_{12, j} r_{12, q} \int V\left(\mathbf{a}_{1}, \mathbf{u}_{12}, \mathbf{a}_{2}\right) f\left(\mathbf{a}_{1} \cdot \mathbf{n}_{1}\right) f\left(\mathbf{a}_{2} \cdot \mathbf{n}_{2}\right) d \mathbf{a}_{1} d \mathbf{a}_{2},\right)\right]_{\mathbf{n}_{2}=\mathbf{n}_{1}=\mathbf{n}},
\end{aligned}
$$

where we have taken into account that according to (22) and (23)

$$
\int d \mathbf{r}_{12} r_{12, j} r_{12, q} \int V\left(\mathbf{a}_{1}, \mathbf{u}_{12}, \mathbf{a}_{2}\right) f\left(\mathbf{a}_{1} \cdot \mathbf{n}_{1}\right) f\left(\mathbf{a}_{2} \cdot \mathbf{n}_{2}\right) d \mathbf{a}_{1} d \mathbf{a}_{2}=\sum_{l m k} S_{l} S_{k} U_{l m k}\left(r_{12}\right) T^{l m k}\left(\mathbf{n}_{1}, \mathbf{u}_{12}, \mathbf{n}_{2}\right) .
$$

One notes that in (B3) the derivative $\partial^{2} /\left(\partial n_{2, t} \partial n_{1, s}\right)$ is acting only on the orientational distribution functions $f\left(\mathbf{a}_{1} \cdot \mathbf{n}_{1}\right)$ and $f\left(\mathbf{a}_{2} \cdot \mathbf{n}_{2}\right)$. Taking into consideration also that

$$
\left[\frac{\partial^{2}}{\partial n_{2, t} \partial n_{1, s}} f\left(\mathbf{a}_{1} \cdot \mathbf{n}_{1}\right) f\left(\mathbf{a}_{2} \cdot \mathbf{n}_{2}\right)\right]_{\mathbf{n}_{2}=\mathbf{n}_{1}=\mathbf{n}}=a_{1, s} a_{2, t} \frac{\partial f\left(\mathbf{a}_{1} \cdot \mathbf{n}\right)}{\partial\left(\mathbf{a}_{1} \cdot \mathbf{n}\right)} \frac{\partial f\left(\mathbf{a}_{2} \cdot \mathbf{n}\right)}{\partial\left(\mathbf{a}_{2} \cdot \mathbf{n}\right)}
$$

allows expressing the tensor (B3) of the nematic phase in the molecular-field approximation in the form:

$$
M_{i q t s}^{\mathrm{MF}}=-\frac{\rho^{2}}{2 k_{B} T} \int r_{12, j} r_{12, q} V\left(\mathbf{a}_{1}, \mathbf{r}_{12}, \mathbf{a}_{2}\right) a_{1, s} a_{2, t} \times \frac{\partial f\left(\mathbf{a}_{1} \cdot \mathbf{n}\right)}{\partial\left(\mathbf{a}_{1} \cdot \mathbf{n}\right)} \frac{\partial f\left(\mathbf{a}_{2} \cdot \mathbf{n}\right)}{\partial\left(\mathbf{a}_{2} \cdot \mathbf{n}\right)} d \mathbf{r}_{12} d \mathbf{a}_{1} d \mathbf{a}_{2} .
$$


This exactly coincides with the general result of Poniewirski and Stecki (B2) if the direct correlation function $C_{2}\left(\mathbf{a}_{1}, \mathbf{r}_{12}, \mathbf{a}_{2}\right)$ is replaced by $-V\left(\mathbf{a}_{1}, \mathbf{r}_{12}, \mathbf{a}_{2}\right) / k_{B} T$ in the molecular-field approximation.

\section{APPENDIX C: CONTRIBUTIONS FROM VARIOUS SPHERICAL INVARIANTS TO THE ELASTIC CONSTANTS}

Let us separately consider the contributions to the elasticity tensor of different terms in Eq. (36). For the invariant $T^{202}$ one obtains

$$
\left[\frac{\partial^{2} T^{202}}{\partial n_{2, t} \partial n_{1, s}}\left(\mathbf{n}_{1}, \frac{\mathbf{r}}{r}, \mathbf{n}_{2}\right)\right]_{\mathbf{n}_{2}=\mathbf{n}_{1}=\mathbf{n}}=3 \delta_{t s}+3 n_{t} n_{s},
$$

and one can readily see that the contribution of the second term to the elasticity tensor Eq. (35) vanishes, while the first term yields the contribution:

$$
\begin{aligned}
K_{i j p q}^{202} & =-\frac{3}{4} \rho^{2} S^{2} \epsilon_{t v i} \epsilon_{t u p} n_{v} n_{u} \int d \mathbf{r} r_{j} r_{q} U_{202}(r) \\
& =-\pi \rho^{2} S^{2} \mathcal{U}^{202} \delta_{j q}\left(\delta_{i p}-n_{i} n_{p}\right)
\end{aligned}
$$

where the constant $\mathcal{U}^{202}$ as well as all similar ones can be evaluated by integrating the potential as in Eq. (A18).

Substituting Eq. (C2) into Eqs. (18)-(20) one obtains that this part of the potential equally contributes to all elastic constants:

$$
K_{11}^{202}=K_{22}^{202}=K_{33}^{202}=-2 \pi \rho^{2} S^{2} \mathcal{U}^{202} .
$$

In a similar way, the derivative of the invariant $T^{404}$ can be expressed as

$$
\left[\frac{\partial^{2} T^{404}}{\partial n_{2, t} \partial n_{1, s}}\left(\mathbf{n}_{1}, \frac{\mathbf{r}}{r}, \mathbf{n}_{2}\right)\right]_{\mathbf{n}_{2}=\mathbf{n}_{1}=\mathbf{n}}=10 \delta_{t s}+45 n_{t} n_{s} .
$$

Equation (C4) is similar to Eq. (C1) and hence one may directly conclude that the $T^{404}$ term in the interaction potential also yields the same contribution to all three elastic constants:

$$
K_{11}^{404}=K_{22}^{404}=K_{33}^{404}=-\frac{20}{3} \pi \rho^{2} S_{4}^{2} \mathcal{U}^{404} .
$$

One notes that the invariants $T^{220}$ and $T^{022}$ depend only on one of the directors, $\mathbf{n}_{1}$ or $\mathbf{n}_{2}$, and hence they do not contribute to the elasticity as their second derivatives in (35) vanish.

To calculate the contributions of the invariants $T^{222}, T^{422}$, and $T^{224}$ we first calculate the contributions from the following different terms: $\left(\mathbf{n}_{1} \cdot \mathbf{n}_{2}\right)\left(\mathbf{n}_{1} \cdot \mathbf{u}\right)\left(\mathbf{n}_{2} \cdot \mathbf{u}\right),\left(\mathbf{n}_{1} \cdot \mathbf{n}_{2}\right)^{2}\left(\mathbf{n}_{2} \cdot\right.$ $\mathbf{u})^{2}$, and $\left(\mathbf{n}_{1} \cdot \mathbf{n}_{2}\right)^{2}\left(\mathbf{n}_{1} \cdot \mathbf{u}\right)^{2}$ :

$$
\begin{aligned}
\int & {\left[\frac{\partial^{2}\left(\mathbf{n}_{1} \cdot \mathbf{n}_{2}\right)\left(\mathbf{n}_{1} \cdot \mathbf{u}\right)\left(\mathbf{n}_{2} \cdot \mathbf{u}\right)}{\partial n_{2 t} \partial n_{1 s}}\right]_{\mathbf{n}_{2}=\mathbf{n}_{1}=\mathbf{n}} u_{j} u_{q} d^{2} \mathbf{u} } \\
= & \int\left[(\mathbf{n} \cdot \mathbf{u})^{2} \delta_{t s}+(\mathbf{n} \cdot \mathbf{u})\left(n_{s} u_{t}+n_{t} u_{s}\right)+u_{s} u_{t}\right] u_{j} u_{q} d^{2} \mathbf{u} \\
= & \frac{4 \pi}{15}\left[2 \delta_{s t}\left(\delta_{j q}+n_{j} n_{q}\right)+2 \delta_{j q} n_{s} n_{t}+\delta_{t j}\left(\delta_{s q}+n_{s} n_{q}\right)\right. \\
& \left.+\delta_{t q}\left(\delta_{s j}+n_{s} n_{j}\right)+\delta_{s j} n_{t} n_{q}+\delta_{s q} n_{j} n_{t}\right],
\end{aligned}
$$

$$
\begin{aligned}
\int & {\left[\frac{\partial^{2}\left(\mathbf{n}_{1} \cdot \mathbf{n}_{2}\right)^{2}\left(\mathbf{n}_{2} \cdot \mathbf{u}\right)^{2}}{\partial n_{2 t} \partial n_{1 s}}\right]_{\mathbf{n}_{2}=\mathbf{n}_{1}=\mathbf{n}} u_{j} u_{q} d^{2} \mathbf{u} } \\
= & \int\left[2(\mathbf{n} \cdot \mathbf{u})^{2}\left(\delta_{s t}+n_{s} n_{t}\right)+4 n_{s} u_{t}(\mathbf{n} \cdot \mathbf{u})\right] u_{j} u_{q} d^{2} \mathbf{u} \\
= & \frac{8 \pi}{15}\left[\left(\delta_{s t}+n_{s} n_{t}\right)\left(\delta_{j q}+2 n_{j} n_{q}\right)+2 \delta_{j q} n_{s} n_{t}\right. \\
& \left.+2 \delta_{t q} n_{s} n_{j}+2 \delta_{t j} n_{s} n_{q}\right], \\
\int & {\left[\frac{\partial^{2}\left(\mathbf{n}_{1} \cdot \mathbf{n}_{2}\right)^{2}\left(\mathbf{n}_{1} \cdot \mathbf{u}\right)^{2}}{\partial n_{2 t} \partial n_{1 s}}\right]_{\mathbf{n}_{2}=\mathbf{n}_{1}=\mathbf{n}} u_{j} u_{q} d^{2} \mathbf{u} } \\
= & \int\left[2(\mathbf{n} \cdot \mathbf{u})^{2}\left(\delta_{s t}+n_{s} n_{t}\right)+4 n_{t} u_{s}(\mathbf{n} \cdot \mathbf{u})\right] u_{j} u_{q} d^{2} \mathbf{u} \\
= & \frac{8 \pi}{15}\left[\left(\delta_{s t}+n_{s} n_{t}\right)\left(\delta_{j q}+2 n_{j} n_{q}\right)+2 \delta_{j q} n_{s} n_{t}\right. \\
& \left.+2 \delta_{s q} n_{t} n_{j}+2 \delta_{s j} n_{t} n_{q}\right] .
\end{aligned}
$$

Note that the integrals over $\mathbf{u}$ are evaluated using a helpful identity:

$$
\int u_{j} u_{q} u_{v} u_{\rho} d^{2} \mathbf{u}=\frac{4 \pi}{15}\left(\delta_{j q} \delta_{v \rho}+\delta_{j \rho} \delta_{v q}+\delta_{j v} \delta_{q \rho}\right) .
$$

Accordingly,

$$
\begin{aligned}
\int & d \mathbf{u} u_{j} u_{q}\left[\frac{\partial^{2} T^{222}}{\partial n_{2, t} \partial n_{1, s}}\left(\mathbf{n}_{1}, \frac{\mathbf{r}}{r}, \mathbf{n}_{2}\right)\right]_{\mathbf{n}_{2}=\mathbf{n}_{1}=\mathbf{n}} \\
= & \frac{2 \pi}{5}\left[-4 \delta_{j q}\left(\delta_{s t}+n_{s} n_{t}\right)+6 \delta_{s t} n_{j} n_{q}+3 \delta_{t j}\left(\delta_{s q}+n_{s} n_{q}\right)\right. \\
& \left.+3 \delta_{t q}\left(\delta_{s j}+n_{s} n_{j}\right)+3 \delta_{s j} n_{t} n_{q}+3 \delta_{s q} n_{j} n_{t}\right]
\end{aligned}
$$

and

$$
\begin{aligned}
K_{i j p q}^{222}= & -\frac{1}{4} \rho^{2} S^{2} \int d r r^{4} U_{222}(r) \epsilon_{t v i} \epsilon_{s u p} n_{v} n_{u} \\
& \times \int d \mathbf{u} u_{j} u_{q}\left[\frac{\partial^{2} T^{222}}{\partial n_{2, t} \partial n_{1, s}}\left(\mathbf{n}_{1}, \frac{\mathbf{r}}{r}, \mathbf{n}_{2}\right)\right]_{\mathbf{n}_{2}=\mathbf{n}_{1}=\mathbf{n}} \\
= & -\frac{\pi}{10} \rho^{2} S^{2} \mathcal{U}^{222}\left[2\left(\delta_{i p}-n_{i} n_{p}\right)\left(3 n_{j} n_{q}-2 \delta_{j q}\right)\right. \\
& \left.+3\left(\epsilon_{q u p} \epsilon_{j v i}+\epsilon_{j u p} \epsilon_{q v i}\right) n_{v} n_{u}\right] .
\end{aligned}
$$

Substituting Eq. (C11) into Eqs. (18)-(20) one finally obtains the following contributions of the $T^{222}$ term to the elastic constants:

$$
\begin{aligned}
K_{11}^{222} & =-\frac{2 \pi}{5} \rho^{2} S^{2} \mathcal{U}^{222}, \\
K_{22}^{222} & =\frac{4 \pi}{5} \rho^{2} S^{2} \mathcal{U}^{222}, \\
K_{33}^{222} & =-\frac{2 \pi}{5} \rho^{2} S^{2} \mathcal{U}^{222} .
\end{aligned}
$$

The second derivative of the function $T^{422}$ can be expressed as:

$$
\begin{gathered}
\int d \mathbf{u} u_{j} u_{q}\left[\frac{\partial^{2} T^{422}}{\partial n_{2, t} \partial n_{1, s}}\left(\mathbf{n}_{1}, \frac{\mathbf{r}}{r}, \mathbf{n}_{2}\right)\right]_{\mathbf{n}_{2}=\mathbf{n}_{1}=\mathbf{n}} \\
=\frac{2 \pi}{3}\left[-\delta_{s t} \delta_{j q}-\delta_{t j} \delta_{s q}-\delta_{t q} \delta_{s j}+5 \delta_{s t} n_{j} n_{q}\right.
\end{gathered}
$$



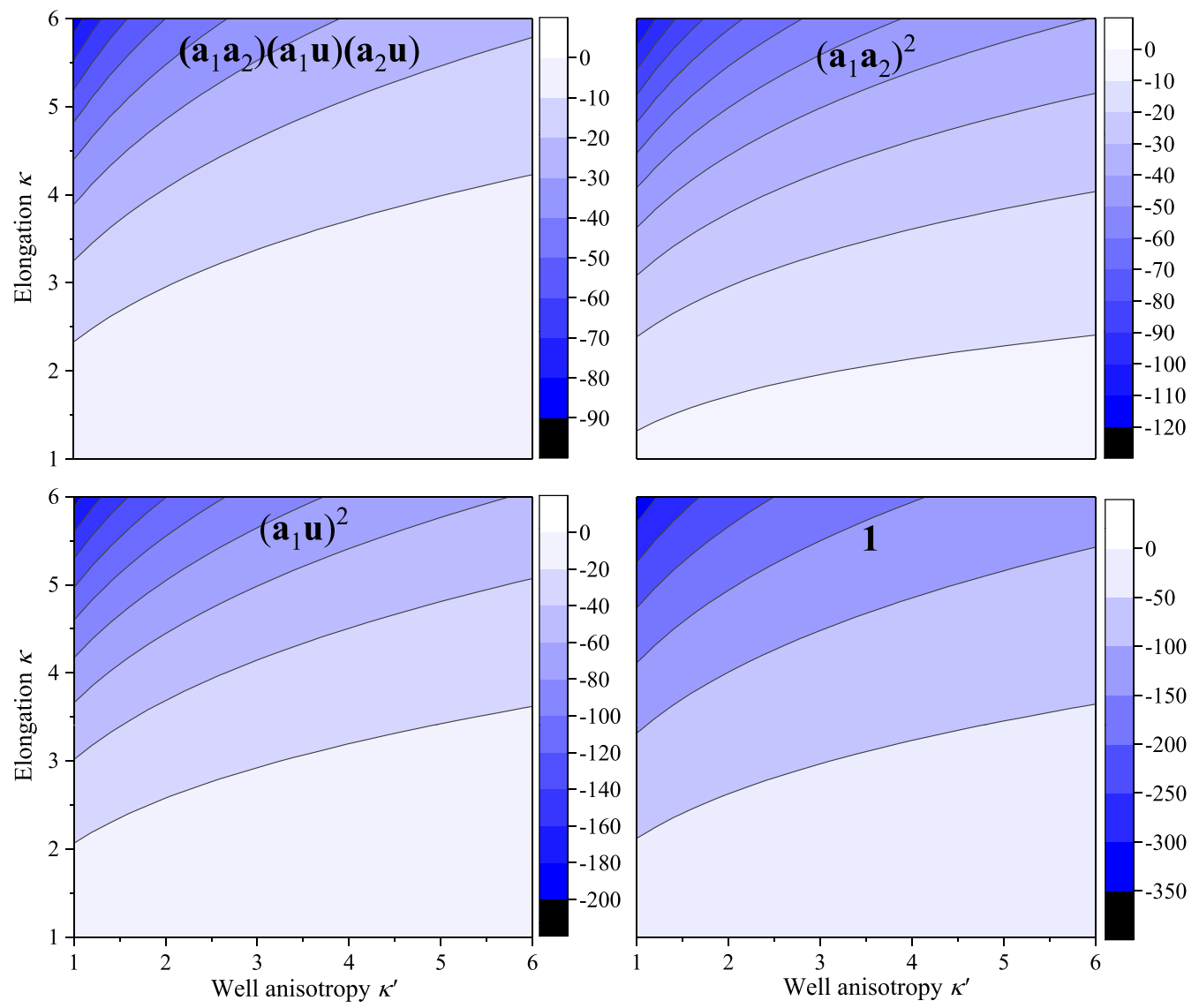

FIG. 10. Colourmaps of the contributions from different vector combinations to the dimensionless parameter $u^{222}$ of the classical GayBerne pair potential with $\mu=2$ and $v=1$.

$$
\begin{aligned}
& +6 \delta_{s q} n_{t} n_{j}+6 \delta_{s j} n_{t} n_{q}+6 \delta_{j q} n_{s} n_{t} \\
& \left.-\delta_{t j} n_{s} n_{q}-\delta_{t q} n_{s} n_{j}+7 n_{s} n_{t} n_{j} n_{q}\right]
\end{aligned}
$$

and the corresponding contribution to the elastic tensor reads:

$$
\begin{aligned}
K_{i j p q}^{422}= & -\frac{1}{4} \rho^{2} S S_{4} \int d r r^{4} U_{422}(r) \epsilon_{t v i} \epsilon_{s u p} n_{v} n_{u} \\
& \times \int d \mathbf{u} u_{j} u_{q}\left[\frac{\partial^{2} T^{422}}{\partial n_{2, t} \partial n_{1, s}}\left(\mathbf{n}_{1}, \frac{\mathbf{r}}{r}, \mathbf{n}_{2}\right)\right]_{\mathbf{n}_{2}=\mathbf{n}_{1}=\mathbf{n}} \\
= & -\frac{\pi}{6} \rho^{2} S S_{4} \mathcal{U}^{422} \epsilon_{t v i} \epsilon_{s u p} n_{v} n_{u} \\
& \times\left[-\delta_{s t} \delta_{j q}-\delta_{t j} \delta_{s q}-\delta_{t q} \delta_{s j}+5 \delta_{s t} n_{j} n_{q}\right] \\
= & \frac{\pi}{6} \rho^{2} S S_{4} \mathcal{U}^{422}\left[\left(\delta_{i p}-n_{i} n_{p}\right)\left(\delta_{j q}-5 n_{j} n_{q}\right)\right. \\
& \left.+\left(\epsilon_{j v i} \epsilon_{q u p}+\epsilon_{q v i} \epsilon_{j u p}\right) n_{v} n_{u}\right],
\end{aligned}
$$

which contributes to the elastic constants (18)-(20) as:

$$
\begin{gathered}
K_{11}^{422}=\pi \rho^{2} S S_{4} \mathcal{U}^{422}, \\
K_{22}^{422}=\frac{\pi}{3} \rho^{2} S S_{4} \mathcal{U}^{422}, \\
K_{33}^{422}=-\frac{4 \pi}{3} \rho^{2} S S_{4} \mathcal{U}^{422} .
\end{gathered}
$$

One can readily see that the contribution of the function $T^{224}$ is the same as that of $T^{422}$ and hence $K_{11}^{224}=$
$K_{11}^{422}, K_{22}^{224}=K_{22}^{422}$, and $K_{33}^{224}=K_{33}^{422}$. Combining the contributions from all invariants one obtains the final expressions for the elastic constants given by Eqs. (41)-(43).

\section{APPENDIX D: CONTRIBUTIONS TO $u^{222}$}

Relatively large positive parameter $u^{222}$ of the original G-B potential with $\mu=2$ and $v=1$ negatively contributes to the elastic constants and destabilizes the nematic phase for $\kappa$ exceeding 2-3. In order to understand the role of different contributions to $u^{222}$, we express it as

$$
\begin{aligned}
u^{222}= & \frac{25}{56 \pi^{2}} \int d \mathbf{a}_{1} d \mathbf{a}_{2} \frac{4 \varepsilon\left(\mathbf{a}_{1}, \mathbf{u}, \mathbf{a}_{2}\right)}{2310 \varepsilon_{0}} \\
& \times\left[461+455 \sigma\left(\mathbf{a}_{1}, \mathbf{u}, \mathbf{a}_{2}\right)+434 \sigma^{2}\left(\mathbf{a}_{1}, \mathbf{u}, \mathbf{a}_{2}\right)\right. \\
& \left.+378 \sigma^{3}\left(\mathbf{a}_{1}, \mathbf{u}, \mathbf{a}_{2}\right)+252 \sigma^{4}\left(\mathbf{a}_{1}, \mathbf{u}, \mathbf{a}_{2}\right)\right] \\
& \times\left[\frac{9}{2}\left(\mathbf{a}_{1} \cdot \mathbf{a}_{2}\right)\left(\mathbf{a}_{2} \cdot \mathbf{u}_{12}\right)\left(\mathbf{a}_{1} \cdot \mathbf{u}_{12}\right)\right. \\
& \left.-\frac{3}{2}\left(\mathbf{a}_{1} \cdot \mathbf{a}_{2}\right)^{2}-\frac{3}{2}\left(\mathbf{a}_{1} \cdot \mathbf{u}_{12}\right)^{2}-\frac{3}{2}\left(\mathbf{u}_{12} \cdot \mathbf{a}_{2}\right)^{2}+1\right]
\end{aligned}
$$

and calculate separately the contributions of the vector combinations in the latter square brackets. As seen in Fig. 10, all 
such contributions are negative and their absolute values are much larger than the resulting parameter $u^{222}$. Therefore, we conclude that this and other more moderate parameters of the classical G-B potential arise as a result of substantial compen- sation of much larger terms. Note, for another example, that the parameter $u^{202}$ stays between -1 and 3, while the separate contributions from $\left(\mathbf{a}_{1} \cdot \mathbf{a}_{2}\right)^{2}$ and unity are larger by orders of magnitude.
[1] P. de Gennes and J. Prost, The Physics of Liquid Crystals (Clarendon Press, London, 1993).

[2] L. M. Blinov, Structure and Properties of Liquid Crystals (Springer Netherlands, Amsterdam, 2011).

[3] V. Borshch, Y.-K. Kim, J. Xiang, M. Gao, A. Jákli, V. P. Panov, J. K. Vij, C. T. Imrie, M. G. Tamba, G. H. Mehl, and O. D. Lavrentovich, Nematic twist-bend phase with nanoscale modulation of molecular orientation, Nat. Commun. 4, 2635 (2013).

[4] K. Adlem, M. Čopič, G. R. Luckhurst, A. Mertelj, O. Parri, R. M. Richardson, B. D. Snow, B. A. Timimi, R. P. Tuffin, and D. Wilkes, Chemically induced twist-bend nematic liquid crystals, liquid crystal dimers, and negative elastic constants, Phys. Rev. E 88, 022503 (2013).

[5] C.-J. Yun, M. R. Vengatesan, J. K. Vij, and J.-K. Song, Hierarchical elasticity of bimesogenic liquid crystals with twist-bend nematic phase, Appl. Phys. Lett. 106, 173102 (2015).

[6] S. Srigengan, M. Nagaraj, A. Ferrarini, R. Mandle, S. J. Cowling, M. A. Osipov, G. Pajak, J. W. Goodby, and H. F. Gleeson, Anomalously low twist and bend elastic constants in an oxadiazole-based bent-core nematic liquid crystal and its mixtures: Contributions of spontaneous chirality and polarity, J. Mater. Chem. C 6, 980 (2018).

[7] S. M. Shamid, S. Dhakal, and J. V. Selinger, Statistical mechanics of bend flexoelectricity and the twist-bend phase in bent-core liquid crystals, Phys. Rev. E 87, 052503 (2013).

[8] M. A. Osipov and G. Pajak, Effect of polar intermolecular interactions on the elastic constants of bent-core nematics and the origin of the twist-bend phase, Eur. Phys. J. E 39, 45 (2016).

[9] M. A. Osipov and G. Pająk, Polar interactions between bentcore molecules as a stabilising factor for inhomogeneous nematic phases with spontaneous bend deformations, Liq. Cryst. 44, 58 (2017)

[10] J. Nehring and A. Saupe, On the elastic theory of uniaxial liquid crystals, J. Chem. Phys. 54, 337 (1971).

[11] R. G. Priest, Theory of the frank elastic constants of nematic liquid crystals, Phys. Rev. A 7, 720 (1973).

[12] J. P. Straley, Frank elastic constants of the hard-rod liquid crystal, Phys. Rev. A 8, 2181 (1973).

[13] A. M. Somoza and P. Tarazona, Frank elastic constants of a nematic liquid crystal of hard molecules, Phys. Rev. A 40, 6069 (1989).

[14] A. Somoza and P. Tarazona, Density functional theory of the elastic constants of a nematic liquid crystal, Mol. Phys. 72, 911 (1991).

[15] M. Osipov and S. Hess, The elastic constants of nematic and nematic discotic liquid crystals with perfect local orientational order, Mol. Phys. 78, 1191 (1993).

[16] W. M. Gelbart and A. Ben-Shaul, Molecular theory of curvature elasticity in nematic liquids, J. Chem. Phys. 77, 916 (1982).

[17] Y. Singh and K. Singh, Density-functional theory of curvature elasticity in nematic liquids. I, Phys. Rev. A 33, 3481 (1986).
[18] K. Singh and Y. Singh, Density-functional theory of curvature elasticity in nematic liquids. II. Effect of long-range dispersion interactions, Phys. Rev. A 34, 548 (1986).

[19] S.-D. Lee and R. B. Meyer, Computations of the phase equilibrium, elastic constants, and viscosities of a hard-rod nematic liquid crystal, J. Chem. Phys. 84, 3443 (1986).

[20] Y. Singh, S. Singh, and K. Rajesh, Molecular theory of elastic constants of liquid crystals: Application to uniaxial phases, Phys. Rev. A 45, 974 (1992).

[21] S. Shri, Curvature elasticity in liquid crystals, Phys. Rep. 277, 283 (1996).

[22] A. Poniewierski and J. Stecki, Statistical theory of the elastic constants of nematic liquid crystals, Mol. Phys. 38, 1931 (1979).

[23] J. Stecki and A. Poniewierski, Application of the statistical theory of elastic constants of nematics to spherocylindrical molecules with attractive forces, Mol. Phys. 41, 1451 (1980).

[24] B. Meer, Molecular Models for Cholesteric and Smectic Liquid Crystals, Ph.D. thesis, University of Groningen, 1979.

[25] L. Blum and A. J. Torruella, Invariant expansion for twobody correlations: Thermodynamic functions, scattering, and the Ornstein-Zernike equation, J. Chem. Phys. 56, 303 (1972).

[26] I. W. Stewart, The Static and Dynamic Continuum Theory of Liquid Crystals (Taylor \& Francis, New York, 2004).

[27] M. A. Osipov, Molecular theories of liquid crystals, in Handbook of Liquid Crystals (Wiley-VCH Verlag GmbH, New York, 2014), pp. 40-71.

[28] M. V. Gorkunov, M. A. Osipov, A. Kocot, and J. K. Vij, Molecular model of biaxial ordering in nematic liquid crystals composed of flat molecules with four mesogenic groups, Phys. Rev. E 81, 061702 (2010).

[29] W. M. Gelbart and B. A. Baron, Generalized van der waals theory of the isotropic-nematic phase transition, J. Chem. Phys. 66, 207 (1977).

[30] J. G. Gay and B. J. Berne, Modification of the overlap potential to mimic a linear site-site potential, J. Chem. Phys. 74, 3316 (1981).

[31] E. de Miguel, L. F. Rull, M. K. Chalam, K. E. Gubbins, and F. V. Swol, Location of the isotropic-nematic transition in the Gay-Berne model, Mol. Phys. 72, 593 (1991).

[32] R. Berardi, A. P. J. Emerson, and C. Zannoni, Monte Carlo investigations of a Gay-Berne liquid crystal, J. Chem. Soc., Faraday Trans. 89, 4069 (1993).

[33] G. R. Luckhurst, R. A. Stephens, and R. W. Phippen, Computer simulation studies of anisotropic systems. XIX. mesophases formed by the Gay-Berne model mesogen, Liq. Cryst. 8, 451 (1990).

[34] M. P. Allen, M. A. Warren, M. R. Wilson, A. Sauron, and W. Smith, Molecular dynamics calculation of elastic constants in Gay-Berne nematic liquid crystals, J. Chem. Phys. 105, 2850 (1996). 